\title{
Optimal Closed-loop Deep Brain Stimulation with Multi-Contact Electrodes
}

Gihan Weerasinghe ${ }^{1}$, Benoit Duchet ${ }^{1}$, Christian Bick ${ }^{2,3,4}$, Rafal ${ }_{3}$ Bogacz ${ }^{1}$

${ }^{1}$ MRC Brain Network Dynamics Unit, Nuffield Department of Clinical

Neurosciences, University of Oxford, Oxford, UK.

${ }^{2}$ Oxford Centre for Industrial and Applied Mathematics, Mathematical Institute, University of Oxford, Oxford, UK.

${ }^{3}$ Centre for Systems, Dynamics and Control and Department of Mathematics, University of Exeter, Exeter, UK.

${ }^{4}$ EPSRC Centre for Predictive Modelling in Healthcare, University of Exeter, Exeter, UK.

E-mail: rafal.bogacz@ndcn.ox.ac.uk 


\section{Introduction}

35

Deep brain stimulation (DBS) is an effective treatment for advanced Parkinson's disease (PD) and essential tremor (ET) which involves delivering stimulation through electrodes implanted deep into the brain and targeting regions thought to be implicated in the disease, which in the case of PD is typically the subthalamic nucleus (STN) and for ET the ventral intermediate nucleus (VIM). PD is a common movement disorder caused by the death of dopaminergic neurons in the substantia nigra. Primarily, symptoms manifest as slowness of movement (bradykinesia), muscle stiffness (rigidity) and tremor. ET is purportedly the most common movement disorder, affecting just under $1 \%$ of the world population $[1,2]$ with the main symptom being involuntary shaking most commonly in the upper limbs [3]. Despite its prevalence, the pathophysiology of ET remains elusive, although the cortex, thalamus and cerebellum are all thought to be involved in the disease [2]. Symptoms of these disorders are thought to be due to overly synchronous activity within neural populations. For PD patients, higher power in the beta frequency range $(13-30 \mathrm{~Hz})$ of the local field potential (LFP) measured in the STN has been shown to correlate with motor impairment [4] while thalamic activity in ET patients is strongly correlated with tremor measured using the wrist flexor EMG [5]. It is thought that DBS acts to desynchronise this pathological activity leading to a reduction in the symptom severity.

A typical DBS system consists of a lead, an implantable pulse generator (IPG) and a unit to be operated by the patient. The DBS lead terminates with an electrode, which is typically divided into multiple contacts. Post surgery, clinicians manually tune the various parameters of stimulation, such as the frequency, amplitude and pulse width, in an attempt to achieve optimal therapeutic benefit. Stimulation is then provided constantly, or 'open-loop', according to these parameters. The choice of stimulation frequency in particular is known to be crucial for efficacy with high frequency (HF) DBS (120-180 Hz) being found to be effective for both PD and ET patients [6]. Despite the effectiveness of conventional HF DBS in treating PD and ET, it is believed that improvements to the efficiency and efficacy can be achieved by using more elaborate stimulation patterns informed by mathematical models. Coordinated reset (CR) neuromodulation is an open-loop DBS strategy where brief HF pulse trains are applied through different contacts of a stimulation electrode $[7,8,9,10]$. The efficacy of CR was first demonstrated theoretically, where precisely-timed delivery of HF pulses can be shown to desynchronise a system of coupled oscillators [7]. In practice, CR has been shown to yield both acute and long-lasting benefits in nonhuman primates [8].

Closed-loop stimulation and IPGs with multiple independent current sources are promising new advances in DBS technology. Closed-loop stimulation is a new development in DBS methods which aims to deliver stimulation on the basis of feedback from a patient. There is a growing body of evidence $[11,12,4,13]$ suggesting that closed-loop stimulation has the potential to offer improvements in terms of efficacy, efficiency and reduction in side effects. IPGs with multiple independent current sources 
are the 'cutting-edge' of DBS technology which, unlike their single current source counterparts, allow for current to be delivered independently to each contact. This gives increased control and flexibility over the shape of the electric fields delivered through the electrodes, allowing for more precise targeting of pathological regions and the possibility of delivering more complex potential fields over space, in addition to allowing for the possibility of recording activity from different regions. The use of multiple contacts for DBS, however, naturally leads to increased complexity, as many more stimulation strategies are now possible. This has created the need to better understand how applying DBS through multiple contacts can affect the treatment.

Closed-loop DBS strategies are characterised by their use of a feedback signal to determine when stimulation should be applied. The choice, use and accuracy of this feedback signal therefore plays a crucial role in determining the efficacy of a particular strategy. In the literature, both the LFP and tremor have been used as feedback signals with studies showing that the effects of DBS to be dependent on both the phase and amplitude of the oscillations at the time of stimulation [12, 4]. In adaptive DBS, high frequency stimulation is applied only when the amplitude of oscillations exceeds a certain threshold [4] and in phase-locked DBS stimulation is applied according to the instantaneous phase of the oscillations, which for ET patients corresponds to stimulation at roughly the tremor frequency (typically $\sim 5 \mathrm{~Hz}$ ) [12]. The combined approach of adaptive and phase-locked stimulation has also been investigated in simulation [13].

In our previous work [14], we provided a mathematical basis for the phase and amplitude dependence of DBS. Here, we extend these ideas and introduce adaptive coordinated reset (ACR), which proposes a closed-loop strategy especially suited to multi-contact systems. Our goal is to understand how the effects of multi-contact DBS should depend on the ongoing neural activity measured through each channel. As part of this work, we demonstrate using numerical simulations that a coupled oscillator model is a plausible neural mechanism for generating tremor found in ET patients. Then, on the basis of this, we model the activity of multiple neural populations using a set of oscillators and relate this activity to the pathological oscillations associated with symptom severity in ET and PD. Using a coupled oscillator model we then describe how this activity (and hence the symptom severity) is likely to change when DBS is applied through multiple contacts. The results we present suggest how DBS should be provided through multiple contacts in order to maximally desynchronise neural activity. Using numerical simulation and parameters fitted to ET patients, we then compare our methods to others found in the literature, namely phase-locked stimulation and coordinated reset. The methods we present can be applied in different ways, either using multiple electrodes or single electrodes with multiple contacts. We therefore use the terms 'electrode' and 'contact' synonymously throughout. 


\section{A Model for Single Contact DBS}

\subsection{Phase Synchrony and Oscillations}

In this section, we consider how stimulation with a single electrode acts on a population 116 of oscillators. Here we follow our previous paper [14], which the interested reader may ${ }_{117}$ refer to for a more detailed derivation of the results presented in this section. A list of ${ }_{118}$ frequently used notation is provided in Table 1.

\begin{tabular}{|c||c|}
\hline Parameter & Description \\
\hline$k$ & Coupling constant \\
$\tilde{\sigma}$ & Noise amplitude \\
$\bar{\omega}$ & Mean of natural frequencies \\
$s_{\omega}$ & Standard deviation of natural frequencies \\
$Z$ & Neuronal phase response curve (nPRC) \\
$a$ & Cosine Fourier coefficient of nPRC \\
$b$ & Sine Fourier coefficient of nPRC \\
$\theta$ & Phase of oscillator \\
$\psi$ & Phase of population \\
$\rho$ & Synchrony of population \\
$r$ & Complex order parameter \\
$c$ & Scaling constant for experimental data \\
$S$ & Number of populations \\
$L$ & Number of electrodes neurons \\
$N$ & Number of constraints \\
$J$ & Population weight \\
$\Gamma$ & Electrode-population distance \\
$w$ & Electrode position \\
$d_{\text {norm }}$ & Neuron position \\
$\mathbf{p}^{\prime}$ & Population position \\
$\mathbf{p}$ & Voltage at electrode \\
$\mathbf{P}$ & Voltage at neuron \\
$v^{\prime}$ & Voltage at population \\
$v$ & Charge of electrode \\
$V$ & Charge of neuron \\
$q^{\prime}$ & Charge of population \\
$q$ & Emplitude response for a single population \\
$Q$ & Element of $\tilde{\mathbf{D}}$ \\
$\mathbf{D}$ & Activity to voltage at electrode transformation matrix \\
$\tilde{\mathbf{D}}$ & Electrode to voltage at population transformation matrix \\
$d$ & $\tilde{d}$
\end{tabular}

Table 1. List of frequently used symbols together with their description. 
Our goal in this subsection is to show how the amplitude measured in feedback signals can be related to the synchrony of neural populations. The instantaneous phase $\Psi(t)$ and envelope amplitude $\mathrm{P}(t)$ of a signal $F(t)$ can be obtained using the analytic signal $R(t)$

$$
R(t)=\mathrm{P} e^{i \Psi}=F(t)+i \hat{H}[F(t)],
$$

where $\hat{H}$ denotes the Hilbert transform. We would like to relate this quantity to those ${ }_{120}$ associated with a state of oscillators.

We define the state of $N$ regular spiking neurons to be given by the set of oscillators $\left\{\theta_{1}(t), \theta_{2}(t), \theta_{3}(t) \ldots \theta_{N}(t)\right\}$, which are the phases describing where each neuron is in its firing cycle. The phase synchrony of this system can be measured using the order parameter $r$, defined to be

$$
r=\rho e^{i \psi}=\frac{1}{N} \sum_{n=1}^{N} e^{i \theta_{n}} .
$$

The above definition ensures the magnitude of the order parameter $\rho$ can take values between 0 and 1, representing full desynchrony and full synchrony, respectively. We can transform the state of the system to a signal representing the neural activity using a superposition of cosine functions

$$
f(t)=\operatorname{Re}(r)=\frac{1}{N} \sum_{n=1}^{N} \cos \left[\theta_{n}(t)\right] .
$$

The choice of a cosine function is for mathematical convenience since it corresponds to the real part of (2). In addition to this, the cosine function has a maximum at 0 , and in classic coupled oscillator models, phase 0 corresponds to the phase when neurons produce spikes [15]. Hence post-synaptic potentials in down-stream neurons receiving an input from the modelled population will be a smoothed function of spikes produced in phase 0 , so the cosine function captures key features of such post-synaptic potentials. Using the Euler relation and comparing (3) with the real part of (2) shows

$$
f(t)=\rho \cos (\psi)
$$

We assume here a simple relationship between the neural activity and feedback signals we may measure, for example tremor and the LFP

$$
F(t)=c f(t)
$$

where the experimental signal has now been denoted by $F(t)$. This is reasonable in the case of ET, where thalamic activity is known to be highly correlated to tremor [5]. Inserting Eq. (5) into (1) gives

$$
\mathrm{P} e^{i \Psi}=c\{f(t)+i \hat{H}[f(t)]\} .
$$

Inserting Eq. (3) into Eq. (6) and using the linearity of $\hat{H}$ leads to

$$
\mathrm{P} e^{i \Psi}=\frac{c}{N} \sum_{n=1}^{N}\left\{\cos \left(\theta_{n}\right)+i \hat{H}\left[\cos \left(\theta_{n}\right)\right]\right\} .
$$


Under the reasonable assumption that the time evolution of $\theta_{n}$ is approximately monotonic, it can be shown that [14]

$$
\hat{H}\left[\cos \left(\theta_{n}\right)\right] \simeq \sin \left(\theta_{n}\right)
$$

where ' $\simeq$ ' is used to indicate 'approximately equal to'. Therefore

$$
\mathrm{P} e^{i \Psi}=\frac{c}{N} \sum_{n=1}^{N}\left\{\cos \left(\theta_{n}\right)+i \sin \left(\theta_{n}\right)\right\}=c \rho e^{i \psi} .
$$

Hence, the instantaneous envelope amplitude and phase (the analytic signal) is relatable to the magnitude and phase of the order parameter using

$$
\mathrm{P}=c \rho, \quad \Psi=\psi
$$

In summary, assuming the experimental data and neural activity are related according $\quad 122$ to Eq. (5) and that the phases $\left\{\theta_{n}\right\}$ increase monotonically with time, we can use ${ }_{123}$ the Hilbert transform of the experimental data to relate the envelope amplitude and ${ }_{124}$ instantaneous phase to the magnitude and phase of the order parameter, respectively. $\quad{ }_{125}$

\subsection{Response Curves}

The neuronal phase response curve (nPRC) for a spiking neuron is the change in spike timing due to a perturbation as a function of the inter-spike time. Hansel et al [16] categorised nPRCs into either type I or type II depending on whether a small excitatory (inhibitory) input always advances (delays) a neuron to a next spike or whether it either advances or delays a spike, depending on where the neuron is in its firing cycle, respectively [17]. These effects of inputs can be captured using a simple mathematical function $Z(\theta)$. By mapping where a neuron is in its firing cycle onto a phase variable $\theta \in[0,2 \pi]$, the nPRC describes the change in phase of a single neuron due to a stimulus. More precisely, under the assumption of a weak input $\epsilon U(t)$, the evolution of a single oscillator can be written in terms of a natural frequency $\omega_{0}$ in addition to a response term

$$
\frac{d \theta}{d t}=\omega_{0}+\epsilon U(t) Z(\theta)
$$

A general neuronal nPRC can be expanded as a Fourier series

$$
Z(\theta)=\frac{a_{0}}{2}+\sum_{m=1}^{\infty} a_{m} \cos (m \theta)+\sum_{m=1}^{\infty} b_{m} \sin (m \theta) .
$$

The nPRC type is reflected in the zeroth harmonic $a_{0}$, or the shift, with $\left|a_{0}\right|$ large ${ }_{127}$ and small relative to the other coefficients being indicative of type I and type II ${ }_{128}$ curves, respectively. Phase oscillator models which incorporate the nPRC can be ${ }_{129}$ shown to reproduce the experimentally-known characteristics of a patient's response to 130 stimulation [14], namely that the effects should be both amplitude and phase dependent ${ }_{131}$ 
$[4,12]$. This leads to the concept of the phase response curve (PRC) and the amplitude ${ }_{132}$ response curve (ARC) for feedback signals, such as LFP and tremor, which can be ${ }_{133}$ described by perturbing a population of oscillators and respectively describe changes ${ }_{134}$ in the phase and amplitude of the feedback signal at the point of stimulation. The ${ }_{135}$ instantaneous curves, which are functions of both the phase and amplitude at which ${ }_{136}$ the stimulation is delivered, are not commonly found in experimental analysis due ${ }_{137}$ primarily to the difficulties associated with obtaining a function of two independent ${ }_{138}$ variables from noisy data. It is more common to find the averaged response curves, 139 which are only functions of the phase and are averaged over the amplitude. Such curves ${ }_{140}$ are readily obtainable using standard signal processing techniques and have been used ${ }_{141}$ to characterise a patient's response to stimulation $[18,12,19]$.

\subsection{The Kuramoto Model}

Modelling the effects of DBS generally poses a challenge since the brain networks involved in disorders such as ET (cortico-thalamic circuit) and PD (cortico-basal-ganglia circuit) are complex and it is still debated from which parts of these circuits the pathological oscillations originate $[20,21]$. The task can be made more tractable by considering a simple phenomenological model which does not attempt to explicitly describe the underlying circuits, but rather focuses on general mechanisms leading to the synchronization of neurons. One example of this is the Kuramoto model, $[22,23]$ where the dynamics of neurons are described using a system of homogeneously coupled oscillators, whose phases evolve according to a set of underlying differential equations. Such models are particularly attractive due to their simplicity and explicit dependence on phase, which makes them convenient for describing the effects of phaselocked stimulation. In the previous section we showed that the oscillation data typically measured in experiment can be modelled using an underlying system of oscillators, whose state is described by the set of $N$ phases $\left\{\theta_{n}\right\}$. We can describe the time evolution of this state (for a single population) using the Kuramoto equations, with an additional term describing the effects of stimulation $[22,7]$

$$
\frac{d \theta_{n}}{d t}=\omega_{n}+\frac{k}{N} \sum_{m=1}^{N} \sin \left(\theta_{m}-\theta_{n}\right)+V(t) Z\left(\theta_{n}\right) .
$$

The first term of (13) is the natural frequency $\omega_{n}$ which describes the frequency in the absence of external inputs. The second term describes the coupling between the activity of individual neurons, where $k$ is the coupling constant which controls the strength of coupling between each pair of oscillators and hence their tendency to synchronize. The third term describes the effect of stimulation, where the intensity of stimulation is denoted by $V(t)$. The nPRC denoted by $Z\left(\theta_{n}\right)$, describes a neuron's sensitivity to stimulation at a particular phase and reflects the observation that the effects of stimulation depend on where a neuron is in its firing cycle [24]. Using the definition of 
the order parameter given in Eq. (2), Eq. (13) can be transformed to give

$$
\frac{d \theta_{n}}{d t}=\omega_{n}+k \rho \sin \left(\psi-\theta_{n}\right)+V(t) Z\left(\theta_{n}\right)
$$

In this form, it is clear that each oscillator has a tendency to move towards the ${ }_{144}$ population phase $\psi$ and that the strength of this tendency is controlled by the coupling ${ }_{145}$ parameter $k$. To gain an intuition for this behaviour readers may wish to explore an ${ }_{146}$ online simulation of the model [25].

\subsection{Reduced Kuramoto Model}

In the previous section, we described the dynamics of a finite system of oscillators using the Kuramoto equations given by Eq. (14). In this model, stimulation is described as a perturbation to the phase of an oscillator, with each oscillator experiencing a different effect of stimulation depending on its phase (and determined by $Z(\theta)$ ). Stimulation therefore has the effect of changing the distribution of oscillators and hence the order parameter of the system. Since the order parameter, given by Eq. (2), is determined by both the amplitude and phase of the system, the expectation is that stimulation will lead to a change in both these quantities, which we refer to as the instantaneous amplitude and phase response of the system. To obtain analytical expressions for these quantities, we can consider an infinite system of oscillators satisfying the ansatz of Ott and Antonsen [26, 27]. In our previous work [14], we showed that for a general $\mathrm{nPRC}$ given by Equation (12) and assuming the natural frequencies are Lorentzian distributed with centre $\omega_{0}$ and width $\gamma$, the instantaneous change in the order parameter can be written as

$$
\begin{gathered}
\frac{d r}{d t}=\left(i \omega_{0}-\gamma\right) r+\frac{k r}{2}\left(1-|r|^{2}\right) \\
+\frac{i V(t)}{2}\left\{a_{0} r+\sum_{m=1}^{\infty} a_{m}\left[\left(r^{*}\right)^{m-1}+r^{m+1}\right]+i \sum_{m=1}^{\infty} b_{m}\left[\left(r^{*}\right)^{m-1}-r^{m+1}\right]\right\} .
\end{gathered}
$$

Using this, we can find expressions for the ARC and PRC due to stimulation

$$
\frac{d \rho_{\text {stim }}}{d t}=\frac{V(t)}{2}\left(1-\rho^{2}\right) \sum_{m=1}^{\infty} \rho^{m-1}\left[a_{m} \sin (m \psi)-b_{m} \cos (m \psi)\right],
$$

and

$$
\frac{d \psi_{\text {stim }}}{d t}=\frac{V(t)}{2}\left\{a_{0}+\left(1+\rho^{-2}\right) \sum_{m=1}^{\infty} \rho^{m}\left[a_{m} \cos (m \psi)+b_{m} \sin (m \psi)\right]\right\}
$$

\section{Reproducing Tremor in ET Patients}

We now address the question of whether the Kuramoto model can produce oscillations which are compatible with tremor data from ET patients. To account for random forces which may influence the firing of individual neurons, the Kuramoto model can be 
extended to include a noise term, which we take here to be a Wiener process. The time evolution for $\theta_{n}$ then becomes

$$
d \theta_{n}=\left[\omega_{n}+k \rho \sin \left(\psi-\theta_{n}\right)+V(t) Z\left(\theta_{n}\right)\right] d t+\tilde{\sigma} N(0,1) \sqrt{d t}
$$

where $\tilde{\sigma}$ is the noise amplitude and $N(0,1)$ is a random number sampled from a standard normal distribution. During a simulation, the set of oscillators $\left\{\theta_{n}\right\}$ evolves according to (18) and oscillations can be generated using Equation (3). The oscillation data output from the Kuramoto model (18) depends on the choice of parameters $\left\{\omega_{n}\right\}, k, N$ and $\tilde{\sigma}$. We can characterise these oscillations by using features extracted from the data, which we choose to be: the power spectral density (PSD), the probability density function (PDF) for the amplitude and the PSD of the envelope amplitude. To reproduce the response of a particular patient, we also fit to the averaged PRC $[14,12,28]$ of the patient by adjusting the parameters for the $\mathrm{nPRC}$. These characterisations can be applied to both the experimental and synthetic simulated data from the model. The similarity between features from the simulated and experimental data can then be quantified using least squares, which in turn allows us to quantify the degree of similarity between simulated and experimental oscillation data. By using this similarity measure as a cost function, we can then find the parameters of the Kuramoto model which minimise the cost using optimisation and thus find the parameters which allow the model to produce oscillations similar to experimental data.

The computational cost of the optimisation depends on a number of factors, including the number of parameters. To ensure feasibility and prevent overfitting, we choose a reasonable number of oscillators $N=60$ and sample $\left\{\omega_{n}\right\}$ from a normal distribution with mean $\bar{\omega}$ and standard deviation $s_{\omega}$. We chose to simulate the Kuramoto model with $\left\{\omega_{n}\right\}$ sampled from a normal distribution as opposed to a Lorentzian distribution (which was assumed in the derivation of the response curves) since the long tails of the latter can lead to a non-monotonic evolution for $\psi$, due to sampling small/negative $\omega_{n}$. This can be problematic for the methodologies used to calculate the phase response curves, which require a monotonic evolution for $\psi$.

We fit the Kuramoto model to tremor data [12, 29] from ET patients deemed to have significant response curves [28]. The parameters found through optimisation are provided in Table 2. Figures 1 and 2(a)-(c) show the Kuramoto model is able to fit well to the features taken from the experimental data. Output from the model can be seen in Figure 3 and shows the resulting simulated data to be quite compatible with that found from experiment. The model can be seen to capture the basic properties of the experimental data, but not the more exotic features, such as the sustained periods of lower amplitudes, which are likely due to non-stationarity. Figures 2(d)-(f) show that the fitted model is able to reproduce the amplitude response for patients generally well, although Figure 2(d) does show a noticeable phase shift between the simulated and experimental curves for Patient 1. Overall, our findings suggest that it is reasonable to use the Kuramoto model as a model for tremor in ET patients. It is on this basis that we derive the expressions for the response curves in subsequent sections. A more detailed 
(a)

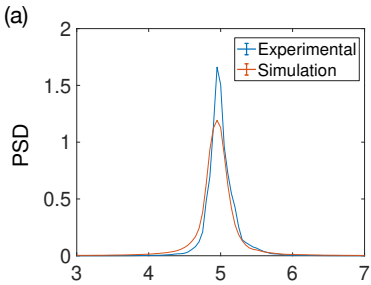

(d)
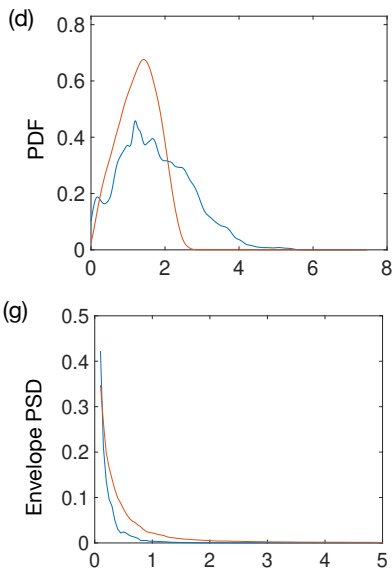

(b)

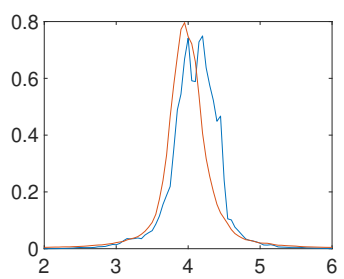

(e)

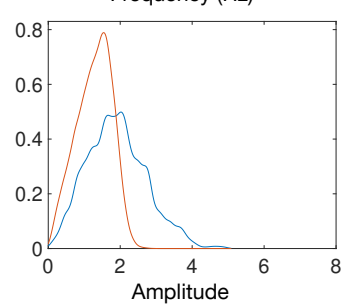

(h)

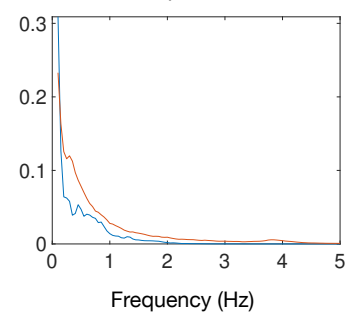

(c)

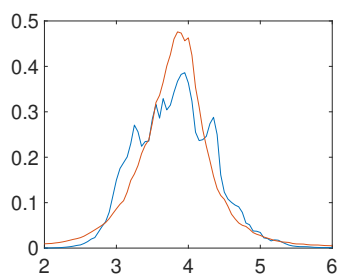

(f)

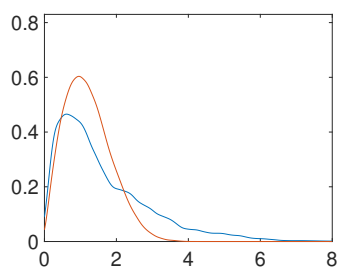

(i)

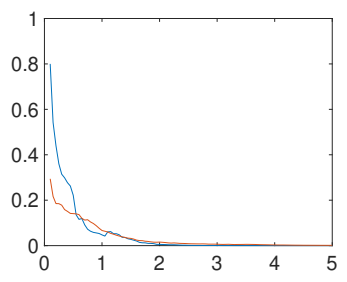

Figure 1. Fits to various features extracted from oscillation data. The first row (a)-(c) is the power spectral density (PSD), the second row (d)-(f) is the probability density function (PDF) for the envelope amplitude and the third row (g)-(i) is the PSD of the envelope. Columns (a)-(g), (b)-(h) and (c)-(i) are for patients 1, 5 and 6, respectively.

\begin{tabular}{|c||c|c|c|c|c||c|c|c|}
\hline Patient & $\tilde{\sigma}$ & $k$ & $\bar{\omega} / 2 \pi$ & $s_{\omega} / 2 \pi$ & $V$ & $a_{0}$ & $a_{1}$ & $b_{1}$ \\
\hline 1 & 2.78 & 9.15 & 4.96 & 0.31 & 0.06 & -0.01 & -0.01 & -0.05 \\
5 & 3.10 & 14.11 & 4.24 & 0.68 & 0.22 & -0.12 & 0.01 & -0.02 \\
6 & 1.58 & 1.57 & 3.88 & 0.44 & 0.15 & -0.07 & -0.02 & 0.01 \\
\hline
\end{tabular}

Table 2. Parameters for the single population Kuramoto model given by Equation (18). The parameters were found by fitting the model to tremor data taken from ET patients by Cagnan et al [12].

description of our fitting methodology, together with details of the experimental data, 188 can be found in the Appendix.

\section{Theory of Multi-contact DBS}

\subsection{Multi-population Kuramoto Model}

We will show in this section that modelling a symptom due to excessive synchrony of multiple neural populations can be achieved by using a simple extension of the concepts presented in Sections 2.1 and 2.3. The set of oscillators $\left\{\theta_{1}(t), \theta_{2}(t), \theta_{3}(t) \ldots \theta_{N}(t)\right\}$ can be arbitrarily divided into $S$ populations with $N_{\sigma}$ oscillators for the $\sigma$ th population. 
(a)

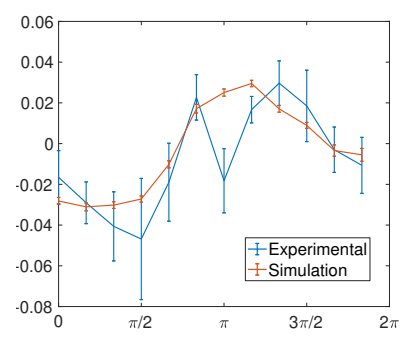

(d)

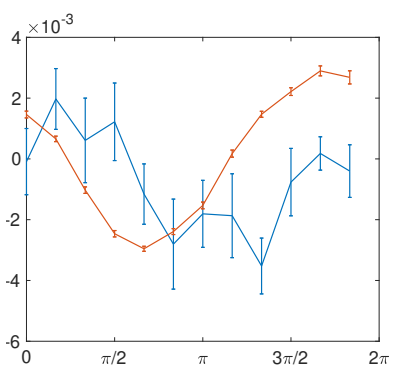

(b)
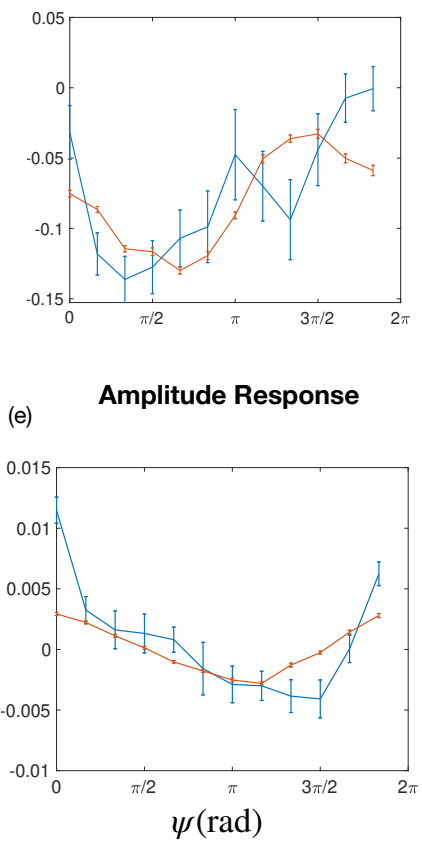

(c)

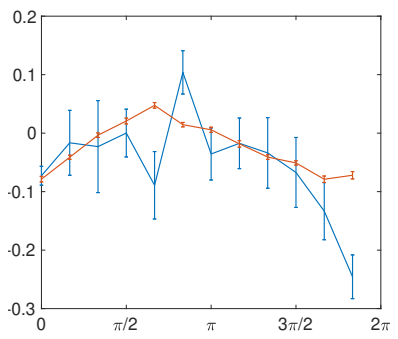

(f)

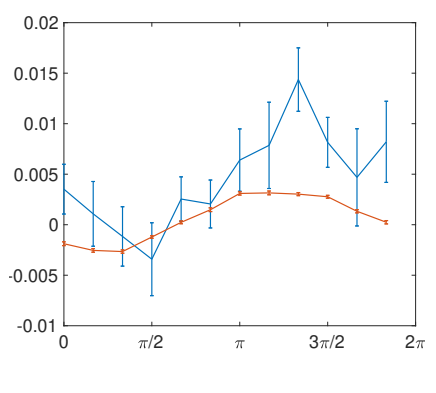

Figure 2. Comparison between the averaged response curves for experimental data and the fitted Kuramoto model. The phase response curve was used as a feature during the fitting procedure. The amplitude response curve is predicted from the model. Columns (a)-(d), (b)-(e) and (c)-(f) are for patients 1, 5 and 6, respectively.

The order parameter defined by Equation (2) can then be rewritten using a double summation

$$
r=\frac{1}{N} \sum_{\sigma=1}^{S} \sum_{n=1}^{N_{\sigma}} e^{i \theta_{\sigma n}}
$$

with oscillator $n$ of population $\sigma$ being denoted by $\theta_{\sigma n}$. The factor of $\frac{1}{N}$ can be brought inside the first summation and rewritten as $\frac{N_{\sigma}}{N_{\sigma} N}$. Then, with

$$
w_{\sigma}=\frac{N_{\sigma}}{N},
$$

the order parameter for the system can be written as

$$
r=\sum_{\sigma=1}^{S} \frac{w_{\sigma}}{N_{\sigma}} \sum_{n=1}^{N_{\sigma}} e^{i \theta_{\sigma n}}
$$

Using the definition of the order parameter (2), Eq. (21) can be written as a weighted superposition of the order parameters for each population

$$
r=\sum_{\sigma=1}^{S} w_{\sigma} r_{\sigma}
$$




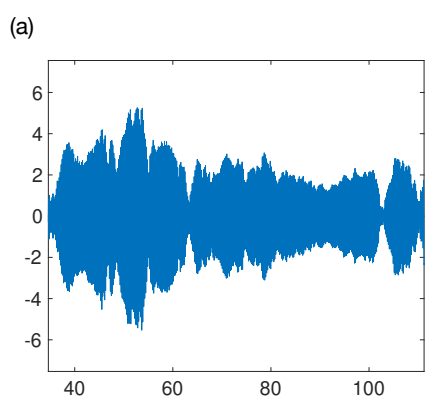

(b)

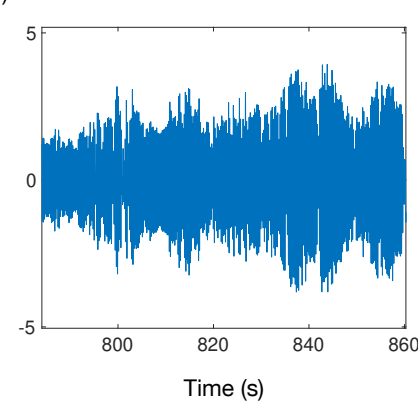

(d)

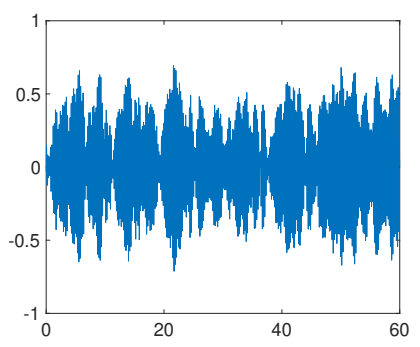

(e)

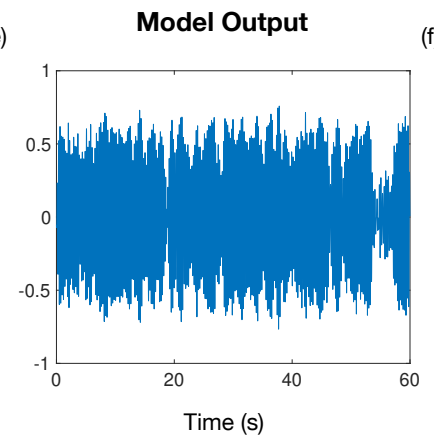

(c)

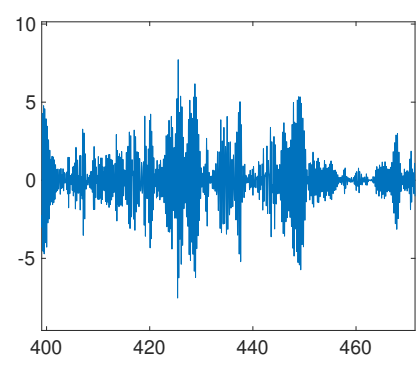

(f)

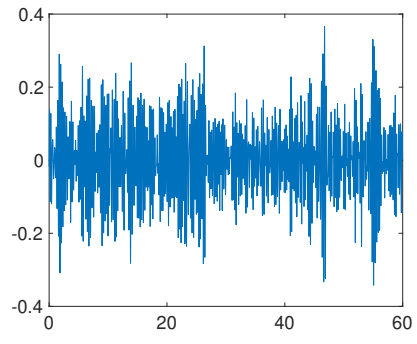

Figure 3. Comparison between experimentally measured tremor data [12] and output from the fitted Kuramoto model. Columns (a)-(d), (b)-(e) and (c)-(f) are for patients 1,5 and 6 , respectively.

with

$$
\sum_{\sigma=1}^{S} w_{\sigma}=1 .
$$

We define $r$ to be the global order parameter with amplitude $\rho$ and phase $\psi$ and $r_{\sigma}$ to ${ }_{192}$ be the local order parameter for population $\sigma$ with amplitude $\rho_{\sigma}$ and phase $\psi_{\sigma}$. The ${ }^{193}$ importance of the global order parameter is that its magnitude $\rho$ is a measure of total ${ }_{194}$ synchrony and hence should be highly correlated to the severity of a symptom, such ${ }_{195}$ as tremor in the case of ET. In the case of PD, symptom severity could be measured ${ }_{196}$ using the unified Parkinson's disease rating scale (UPDRS) scores [30]. Therefore, we ${ }^{197}$ will consider how to stimulate to maximally reduce the magnitude of the global order ${ }_{198}$ parameter.

We can also relate (22) to feedback signals we might measure by using (3) and taking the real part. Under the assumption (5) relating the neural activity to the feedback signal we obtain an expression for the feedback signal in terms of population activities

$$
F(t)=\sum_{\sigma=1}^{S} c w_{\sigma} f_{\sigma}(t) .
$$

We refer to $F(t)$ and $\left\{f_{\sigma}(t)\right\}$ as the global and local signals (or population activities), respectively. Using (4), Equation (24) can also be written in terms of the global and 
local amplitudes and phases

$$
\mathrm{P} \cos (\psi)=\sum_{\sigma=1}^{S} c w_{\sigma} \rho_{\sigma} \cos \left(\psi_{\sigma}\right)
$$

The Kuramoto equations (13) can also be rewritten in terms of the population phases $\psi_{\sigma}$ and amplitudes $\rho_{\sigma}$

$$
\frac{d \theta_{\sigma n}}{d t}=\omega_{\sigma n}+\sum_{\sigma^{\prime}=1}^{S} w_{\sigma^{\prime}} k_{\sigma \sigma^{\prime}} \rho_{\sigma^{\prime}} \sin \left(\psi_{\sigma^{\prime}}-\theta_{\sigma n}\right)+V_{\sigma}(t) Z_{\sigma}\left(\theta_{\sigma n}\right),
$$

where $V_{\sigma}(t)$ is the now the stimulation intensity at a population $\sigma$. The coupling constant 200 $k$ in Eq. (13) is now a $S \times S$ matrix with elements $k_{\sigma \sigma^{\prime}}$. The diagonal and off-diagonal 201 elements describe the intrapopulation and interpopulation coupling, respectively.

\subsection{Multi-population Response Curves}

We now derive an expression describing the change in the global amplitude due to stimulation as a function of the local (population) amplitudes and phases. For now it is assumed that the local quantities (to base the stimulation on) can be measured. We will discuss how these quantities can be measured later. Using the polar form of the order parameter (2), Equation (22) can be written as a summation involving the amplitudes and phases of individual populations

$$
\rho e^{i \psi}=\sum_{\sigma=1}^{S} w_{\sigma} \rho_{\sigma} e^{i \psi_{\sigma}}
$$

Taking the time derivative of (27) leads to

$$
\frac{d \rho}{d t}+i \rho \frac{d \psi}{d t}=\sum_{\sigma=1}^{S} w_{\sigma}\left[\frac{d \rho_{\sigma}}{d t}+i \rho_{\sigma} \frac{d \psi_{\sigma}}{d t}\right] e^{i\left(\psi_{\sigma}-\psi\right)}
$$

which can be written in terms of the real and imaginary components

$$
\begin{aligned}
& \frac{d \rho}{d t}+i \rho \frac{d \psi}{d t}=\sum_{\sigma=1}^{S} w_{\sigma}\left\{\left[\frac{d \rho_{\sigma}}{d t} \cos \left(\psi_{\sigma}-\psi\right)-\rho_{\sigma} \frac{d \psi_{\sigma}}{d t} \sin \left(\psi_{\sigma}-\psi\right)\right]\right. \\
& \left.+i\left[\rho_{\sigma} \frac{d \psi_{\sigma}}{d t} \cos \left(\psi_{\sigma}-\psi\right)+\frac{d \rho_{\sigma}}{d t} \sin \left(\psi_{\sigma}-\psi\right)\right]\right\}
\end{aligned}
$$

It can be seen that the time derivative of the amplitude is the real part of (29)

$$
\frac{d \rho}{d t}=\sum_{\sigma=1}^{S} w_{\sigma}\left[\frac{d \rho_{\sigma}}{d t} \cos \left(\psi_{\sigma}-\psi\right)-\rho_{\sigma} \frac{d \psi_{\sigma}}{d t} \sin \left(\psi_{\sigma}-\psi\right)\right] .
$$

The quantities $d \rho_{\sigma} / d t$ and $d \psi_{\sigma} / d t$ of Equation (30) are the changes in the amplitude and phase of a population with respect to time. If we assume the distribution of phases 
(a)

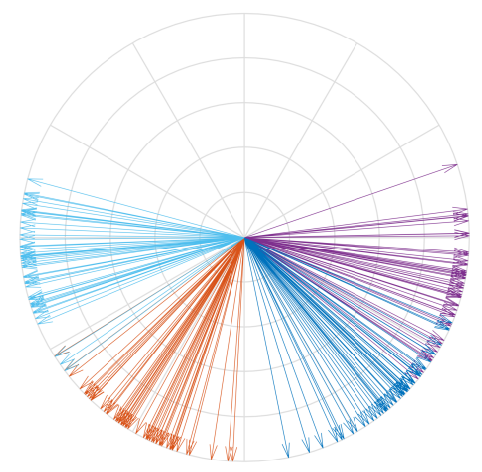

(b)

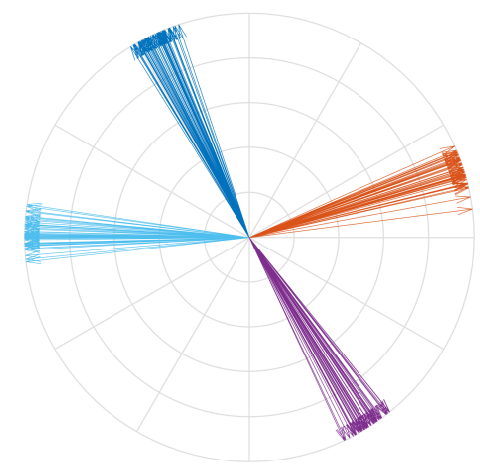

Figure 4. Different configurations of oscillators color coded according to population showing (a) unimodal distribution (b) multimodal (clustered) distribution. Configurations were obtained by simulating the multi-population Kuramoto equations $(26)$.

within a population satisfies the ansatz of Ott and Antonsen [26], we can substitute Eq. (16) and Eq. (17) into (30) to obtain the amplitude response due to stimulation in terms of the Fourier coefficients of $Z(\theta)$

$$
\begin{aligned}
\frac{d \rho_{\text {stim }}}{d t}=\frac{1}{2} \sum_{\sigma=1}^{S} w_{\sigma} V_{\sigma}(t)\{ & \sum_{m=1}^{\infty} \rho_{\sigma}^{m-1}\left[a_{m} \sin \left[(m-1) \psi_{\sigma}+\psi\right]-b_{m} \cos \left[(m-1) \psi_{\sigma}+\psi\right]\right] \\
& \left.-\sum_{m=0}^{\infty} \rho_{\sigma}^{m+1}\left[a_{m} \sin \left[(m+1) \psi_{\sigma}-\psi\right]-b_{m} \cos \left[(m+1) \psi_{\sigma}-\psi\right]\right]\right\},
\end{aligned}
$$

where, for simplicity, we assume that $Z(\theta)$ is the same for all populations. Equation (31) contains an expansion over the harmonics of $Z(\theta)$. In our previous paper, we demonstrated that, for a biologically realistic $\mathrm{nPRC}$, it is reasonable to neglect higher harmonic terms $(m>1)$ [14], leading to a simpler expression for the instantaneous amplitude response

$$
\begin{aligned}
\frac{d \rho_{\mathrm{stim}}}{d t} \simeq \frac{1}{2} \sum_{\sigma=1}^{S} w_{\sigma} V_{\sigma}(t)\left\{\left[a_{1} \sin (\psi)\right.\right. & \left.-b_{1} \cos (\psi)\right]-\rho_{\sigma} a_{0} \sin \left(\psi_{\sigma}-\psi\right) \\
& \left.-\rho_{\sigma}^{2}\left[a_{1} \sin \left(2 \psi_{\sigma}-\psi\right)-b_{1} \cos \left(2 \psi_{\sigma}-\psi\right)\right]\right\},
\end{aligned}
$$

Equation (32) shows the global reduction in amplitude can be expressed as a sum of contributions from each population, with each term dependent on 3 variables: the global phase $\psi$, the local phase $\psi_{\sigma}$ and the local amplitude $\rho_{\sigma}$. It also suggests that stimulating on the basis of local quantities may not always be advantageous. It can be seen that the terms of Equation (32) can be divided into two categories: ones which depends on both global and local quantities and ones which depends only on global quantities. The 
(a)

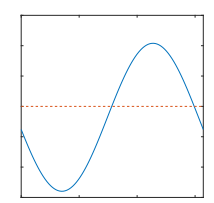

(b)

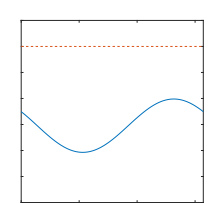

(c)

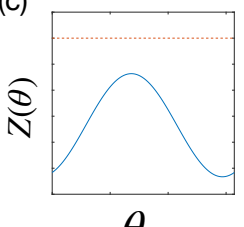

$\rho_{\sigma}=0.20$
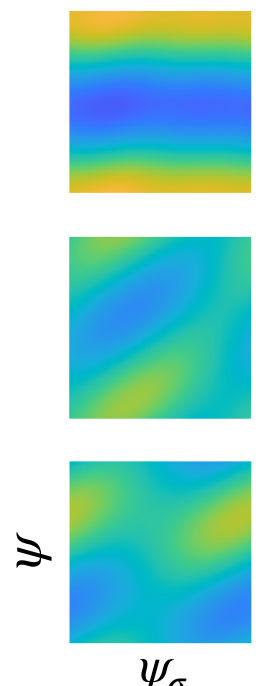
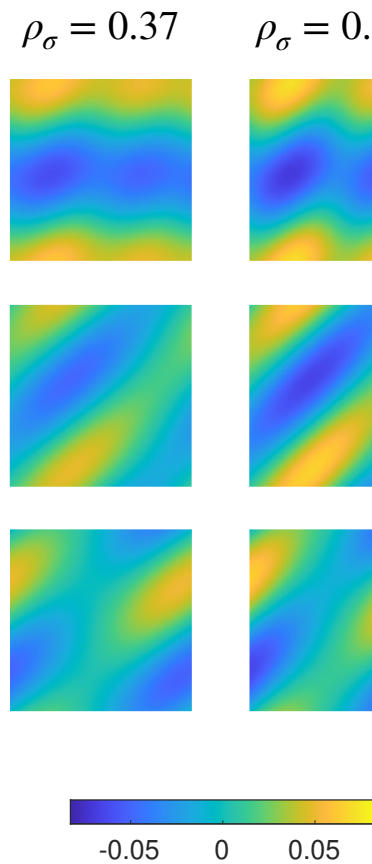
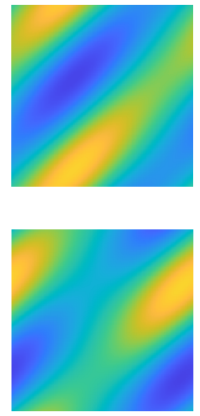

$\rho_{\sigma}=0.53 \quad \rho_{\sigma}=0.70$
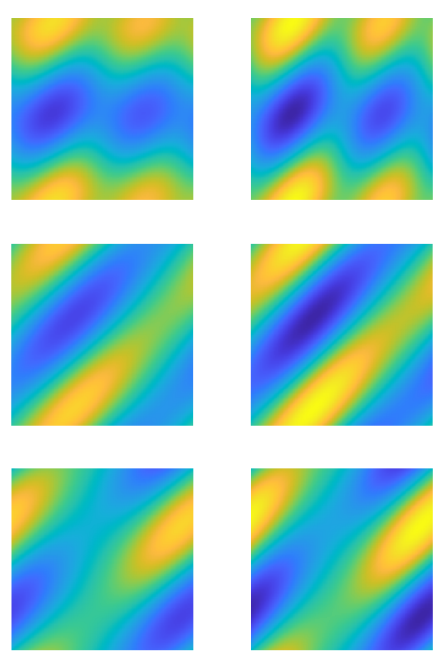

Figure 5. The predicted contribution of a single population to the amplitude response at different local amplitudes $\rho_{\sigma}$ according to Eq. (32). Each panel corresponds to a single ET patient from the study of Cagnan et al [12], where the Fourier coefficients of the nPRC were determined using a fitting procedure. Panels (a) (b) and (c) are for patients 1,5 and 6 , respectively. For each plot, the vertical axis is the global phase $(\psi)$ and the horizontal axis is the local (or population) phase $\left(\psi_{\sigma}\right)$. The corresponding nPRC $Z(\theta)$ is also shown, with zero indicated by a red dashed line. Blue regions indicate areas where stimulation is predicted to suppress amplitude.

terms depending on both the global and local phases are also dependent on the local amplitudes. In cases where the local amplitude is small, i.e. $\rho_{\sigma} \ll 1$, we can neglect the term involving $\rho_{\sigma}^{2}$, leading to a simplified expression

$$
\frac{d \rho_{\text {stim }}}{d t} \simeq \frac{1}{2} \sum_{\sigma=1}^{S} w_{\sigma} V_{\sigma}(t)\left\{\left[a_{1} \sin (\psi)-b_{1} \cos (\psi)\right]-\rho_{\sigma} a_{0} \sin \left(\psi_{\sigma}-\psi\right)\right\} .
$$

Here, it can be seen that the amplitude response would be dependent only on the global ${ }_{204}$ phase if the zeroth harmonic of the nPRC $a_{0}$ is negligible, which is the case for type II ${ }_{205}$ nPRCs. It can also be seen that the dependency of the amplitude response on the local 206 quantities of population $\sigma$ becomes less at increasingly lower local amplitudes $\rho_{\sigma}$. In ${ }_{207}$ addition to this, the dependence on $\sin \left(\psi_{\sigma}-\psi\right)$ implies that stimulating on the basis of ${ }_{208}$ local quantities would only have an effect if the phases of individual populations differ 209 sufficiently from the mean phase. One situation in which such phase difference may be 210 particularly high are for clustered configurations of oscillators. Examples of different ${ }_{211}$ configurations of oscillators are shown in Figure 4. 
Plots for the amplitude response (32) together with the corresponding nPRC using 213 the fitted parameters from Table 2 for ET patients 1, 5 and 6 can be seen in Figures 5(a), ${ }^{214}$ (b) and (c), respectively. For a given local amplitude, we plot a single term from ${ }_{215}$ the summation over populations in Equation (32). This provides the contribution of ${ }_{216}$ a single population to the amplitude response as a function of the local and global ${ }_{217}$ phases. Regions in blue are areas of amplitude suppression while orange regions predict ${ }_{218}$ amplification. In both cases, these regions can be seen to occur in bands. Graphically, ${ }^{219}$ the dependence of the amplitude response on the global and local phases can be inferred 220 from the direction of the banding. A purely horizontal band implies the amplitude 221 response is independent of the local phase. An example of this can be seen at low ${ }_{222}$ amplitudes in Figure 5 (a). Other plots show diagonal banding, which implies the ${ }_{223}$ amplitude response is dependent on both the global and local phases. This behaviour ${ }^{224}$ can be understood by considering the 3 terms of (32). At low amplitudes, the first ${ }_{225}$ term dominates, which is only dependent of the global phase. As the local amplitude ${ }_{226}$ increases, the second and third terms depending on local quantities become increasingly ${ }_{227}$ more important. For the cases where $\left|a_{0}\right|$ is small, the effect is less apparent. The left ${ }_{228}$ panel of Figure 5 (a) shows that stimulation can either increase or reduce the phase (i.e. ${ }^{229}$ an nPRC of type II), implying a relatively small $\left|a_{0}\right|$. Hence, for this patient, the second 230 and third terms are negligible, except at higher amplitudes. Figures 5 (b) and (c) shows 231 that stimulation has the effect of only increasing the phase, which is indicative of $Z(\theta){ }^{232}$ with larger $\left|a_{0}\right|$. For these systems the amplitude response can be seen to depend more ${ }^{233}$ strongly on the local phase for all amplitudes.

\subsection{Obtaining Population Activities Through Electrode Measurements}

235

In this subsection, we will describe how the local phases $\left\{\psi_{\sigma}\right\}$ and amplitudes $\left\{\rho_{\sigma}\right\}$ can be recovered using LFP measurements through different contacts. This requires us to incorporate information about the geometry of the electrode placement into the equations for the response curve in addition to assigning a physical interpretation to the population activity. Our aim here is not to construct a detailed electrophysiological model of neuronal activity but rather to present a very general form for the voltage measured at an electrode contact. We formulate our expressions here in terms of electric charge, but the same form also permits the use of currents. In addition to this, our expressions include summations over neurons, but an equally valid expression can be made by summing over elements of space, as is the case in multi-compartmental models [31]. The quantities we consider in our model are voltages $v_{l}^{\prime}(t)$ measured at electrode $l$ due to the activity of population $\sigma$ producing charges $Q_{\sigma}(t)$ and voltages $V_{\sigma}(t)$ at population $\sigma$ due to stimulation which delivers charge $q_{l}^{\prime}(t)$ to electrode $l$. The voltage $V_{\sigma}(t)$ can also be thought of as the 'stimulation intensity' experienced at population $\sigma$.

We begin by considering a system of $L$ electrodes and $N$ neurons with positions in space denoted by $\mathbf{p}^{\prime}$ and $\mathbf{p}$, respectively. From now on, we will use the following notation throughout: primes to denote quantities associated with electrodes, lower case 
for neuronal quantities and upper case for population quantities. Voltages measured at an electrode arise due to the geometry of the electrode-neuron system and the intrinsic electrical activity of each neuron. We express the voltage measured at an electrode in terms of a summation over charges due to the neurons $q_{n}(t)$

$$
v_{l}^{\prime}(t)=\sum_{n=1}^{N} d\left(\mathbf{p}^{\prime}, \mathbf{p}_{n}\right) q_{n}(t)
$$

where $d\left(\mathbf{p}_{l}^{\prime}, \mathbf{p}_{n}\right)$ are coefficients which reflect the medium and geometry of the electrodeneuron system. For example, in the case of a coulombic system, the coefficients would be

$$
d\left(\mathbf{p}_{l}^{\prime}, \mathbf{p}_{n}\right)=\frac{\kappa_{e}}{\left|\mathbf{p}_{l}^{\prime}-\mathbf{p}_{n}\right|}
$$

where $\kappa_{e}$ is the Coulomb constant. As before, a system of neurons can be arbitrarily divided into $S$ populations, with each neuron referenced by both a population and position index $\sigma$ and $n$, respectively.

$$
v_{l}^{\prime}(t)=\sum_{\sigma=1}^{S} \sum_{n=1}^{N_{\sigma}} d\left(\mathbf{p}_{l}^{\prime}, \mathbf{p}_{\sigma n}\right) q_{\sigma n}(t),
$$

We now let $\mathbf{p}_{\sigma n}=\mathbf{P}_{\sigma}+\Delta \mathbf{p}_{\sigma n}$, i.e. we now define a vector to a neuron in terms of a vector to a region (or population) plus a shift.

$$
v_{l}^{\prime}(t)=\sum_{\sigma=1}^{S} \sum_{n=1}^{N_{\sigma}} d\left(\mathbf{p}_{l}^{\prime}, \mathbf{P}_{\sigma}+\Delta \mathbf{p}_{\sigma n}\right) q_{\sigma n}(t),
$$

If we assume the region at $\mathbf{P}_{\sigma}$ to be small, then

$$
\Delta \mathbf{p}_{\sigma n} \simeq 0
$$

The potential at the electrode can then be written in terms of population activity

$$
v_{l}^{\prime}(t)=\sum_{\sigma=1}^{S} d\left(\mathbf{p}_{l}^{\prime}, \mathbf{P}_{\sigma}\right) Q_{\sigma}(t)
$$

where

$$
Q_{\sigma}(t)=\sum_{n=1}^{N_{\sigma}} q_{\sigma n}(t) .
$$

The time dependent charge of a population $Q_{\sigma}(t)$ can be related to the neural activity by assuming a form for $q_{\sigma n}(t)$, specifically that

$$
q_{\sigma n}(t)=\frac{c}{N} \cos \left(\theta_{\sigma n}\right)
$$

Inserting this into (40) and using (20) gives

$$
Q_{\sigma}(t)=\frac{c w_{\sigma}}{N_{\sigma}} \sum_{n=1}^{N_{\sigma}} \cos \left(\theta_{\sigma n}\right) .
$$


(a)

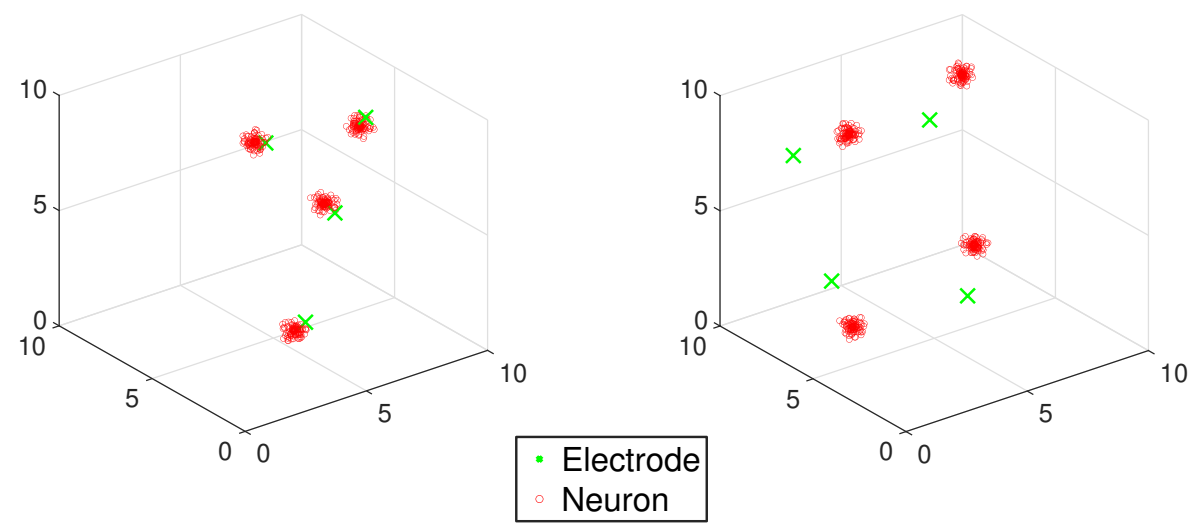

Figure 6. Visualisations of 4 electrode 4 population systems, where each population occupies a small spatial region. Each system was generated by randomly choosing the coordinates of the 4 populations so that they lie within a box of length $L_{\mathrm{box}}=10$. Each electrode is then placed $d_{\text {norm }}$ distance from a population. Panel (a) shows a configuration where each electrode is placed very close to a population $\left(d_{\text {norm }}=0.5\right)$. Panel $(\mathrm{b})$ shows a different system $\left(d_{\text {norm }}=2\right)$ where both the electrodes and populations are more 'dispersed'. In this scenario, electrodes may record activity from multiple populations.

Using (3) and (4) gives an expression for the time dependent charge of a population in terms of the population phase and amplitude

$$
Q_{\sigma}(t)=c w_{\sigma} \rho_{\sigma} \cos \left(\psi_{\sigma}\right)
$$

Using (39) the potential at the electrodes can therefore be written in matrix form

$$
\left(\begin{array}{ccccc}
d_{11} & d_{12} & d_{13} & \ldots & d_{1 S} \\
\vdots & \ddots & & & \\
\vdots & & \ddots & & \\
\vdots & & & \ddots & \\
d_{L 1} & & & & d_{L S}
\end{array}\right)\left(\begin{array}{c}
\rho_{1} \cos \left(\psi_{1}\right) \\
\rho_{2} \cos \left(\psi_{2}\right) \\
\rho_{3} \cos \left(\psi_{3}\right) \\
\vdots \\
\rho_{S} \cos \left(\psi_{S}\right)
\end{array}\right)=\left(\begin{array}{c}
v_{1}^{\prime}(t) \\
v_{2}^{\prime}(t) \\
v_{3}^{\prime}(t) \\
\vdots \\
v_{L}^{\prime}(t)
\end{array}\right),
$$

where for simplicity we have denoted $d_{l \sigma}=c w_{\sigma} d\left(\mathbf{p}_{l}^{\prime}, \mathbf{P}_{\sigma}\right)$. Equation (44) can be expressed in a more compact form with $\mathbf{D}$ denoting the matrix of coefficients (of dimensions $L \times S)$, $\mathbf{f}$ as the vector of neural activities and $\mathbf{v}^{\prime}$ as the vector of electrode measurements.

$$
\mathrm{Df}=\mathbf{v}^{\prime}
$$

Equation (45) relates the voltages at the electrodes $\mathbf{v}^{\prime}$ to the neural activities $\mathbf{f}$. In 250 general, our ability to use Equation (32) in a closed-loop DBS strategy depends on being ${ }^{251}$ able to accurately measure the population quantities $\left\{\rho_{\sigma}\right\}$ and $\left\{\psi_{\sigma}\right\}$. Equation (44) ${ }_{252}$ shows that what we actually measure at the electrodes is a linear superposition of ${ }_{253}$ 
population activities. For the cases where $\mathbf{D}$ is approximately diagonal, the population ${ }^{254}$ quantities could be accurately recovered (although $\rho_{\sigma}$ would be scaled). Such cases 255 would represent systems consisting of small separated regions of activity, with each 256 electrode positioned close to each region (see Figure 6(a)).

Methods such as independent component analysis (ICA) [32] are well-suited to 258 solving the general problem of recovering a vector of 'source signals' $\mathbf{f}(t)$ (in this case the ${ }_{259}$ population activities) given a vector of recordings $\mathbf{v}^{\prime}(t)$, as expressed in Equation (44), 260 although the method cannot recover the scaling. We consider the special case of a single ${ }_{261}$ contact recording, i.e. with $L=1$, in the appendix. Since in theory the matrix $D$ should ${ }_{262}$ not evolve with time, we envisage ICA being applied offline to recover $D$ and then used ${ }^{263}$ to obtain the local signals. In practice, after determining the local signals, Equation (25) 264 should be used to construct the global signal. In this process, the weights $\left\{w_{\sigma}\right\}$ should ${ }_{265}$ be chosen to give a global signal with an amplitude that is highly correlated to the ${ }_{266}$ symptom severity.

\subsection{Optimal Stimulation Strategy}

The equations for the amplitude response (31), (32) and (33) depend on the stimulation ${ }_{269}$ intensity at a population $V_{\sigma}$. It is implied, therefore, that the 'population' exists at 270 some region in space and that $V_{\sigma}$ should take into account the geometry of the electrode placement, how electric fields behave within brain tissue and the charges on a particular electrode. In this subsection, our aim is to incorporate these ideas into an expression for the amplitude response.

Equations (31), (32) and (33) all involve summations over populations, with each term being the product of a weight $w_{\sigma}$, a stimulation intensity $V_{\sigma}$ and some intrinsic response, which we shall denote here by $\Gamma_{\sigma}$. For example, in the case of Equation (31) $\Gamma_{\sigma}$ would be

$$
\begin{aligned}
\Gamma_{\sigma}= & \sum_{m=1}^{\infty} \rho_{\sigma}^{m-1}\left[a_{m} \sin \left[(m-1) \psi_{\sigma}+\psi\right]-b_{m} \cos \left[(m-1) \psi_{\sigma}+\psi\right]\right] \\
& -\sum_{m=0}^{\infty} \rho_{\sigma}^{m+1}\left[a_{m} \sin \left[(m+1) \psi_{\sigma}-\psi\right]-b_{m} \cos \left[(m+1) \psi_{\sigma}-\psi\right]\right],
\end{aligned}
$$

Using this, a more compact expression for the amplitude response can be written using linear algebra notation, with $\boldsymbol{\Gamma}$ equal to the vector of responses and $\mathbf{V}$ equal to the vector of voltages at a population

$$
\frac{d \rho_{\mathrm{stim}}}{d t}=\frac{1}{2}\left(\boldsymbol{\Gamma}^{\top} \mathbf{V}\right)
$$

where the weights $w_{\sigma}$ are now considered as part of the response $\boldsymbol{\Gamma}$. The amplitude response involves a 'stimulation intensity' $\mathbf{V}(t)$ - an abstract quantity which, intuitively, should not only depend on the charge characteristics at the electrode, but also the geometry of the electrode placement and the properties of the brain tissue. Taken 
altogether, the stimulation intensity is better interpreted as the voltage at a population, which can be expressed as a weighted superposition of charges at the electrodes

$$
\tilde{\mathrm{D}} \mathbf{q}^{\prime}=\mathbf{V}
$$

As before, the elements of matrix $\tilde{\mathbf{D}}$ (of dimensions $S \times L$ ) are coefficients which reflect the medium and geometry of the electrode-neuron system. Its worth noting here that Equations (45) and (48) can also be used to model systems where the stimulating and recording electrodes are different, since $\tilde{\mathbf{D}}$ is allowed to be different from $\mathbf{D}^{\top}$. Inserting (48) into (47) leads to an expression for the amplitude response in terms of the charges at the electrodes, i.e. the control variables

$$
\frac{d \rho_{\mathrm{stim}}}{d t}=\frac{1}{2}\left(\tilde{\mathbf{D}}^{\top} \boldsymbol{\Gamma}\right)^{\top} \mathbf{q}^{\prime}
$$

The quantity $\left(\tilde{\mathbf{D}}^{\top} \boldsymbol{\Gamma}\right)^{\top}$ is defined for each time step so that the optimisation becomes a problem of choosing $\mathbf{q}^{\prime}$ so as to minimise $d \rho_{\text {stim }} / d t$. Often, concern for tissue damage due to stimulation imposes a limit on how much charge can be delivered to a single or group of contact(s). To account for this and ensure feasibility, we impose two constraints. The first constraint ensures the charge for a particular contact does not exceed some maximum value $q_{\max }^{\prime}$

$$
0 \leq \mathbf{q}^{\prime} \leq q_{\max }^{\prime}
$$

A simple optimal solution (per time step) for Equations (49) and (50) can be found by setting the charge for the $l$ th contact to $q_{\max }^{\prime}$ if the $l$ th component of $\left(\tilde{\mathbf{D}}^{\top} \boldsymbol{\Gamma}\right)^{\top}$ is negative. A second constraint ensures the charge density within a region does not become dangerously high

$$
A \mathbf{q}^{\prime} \leq \mathbf{q}_{\max }^{\prime}
$$

Here, for $J$ groups of contacts, the constraint matrix A has dimension $J \times L$ and can ${ }_{275}$ be used to constrain the collective charges of the group. The $J$-dimensional vector $\mathbf{q}_{\max }^{\prime}{ }^{276}$ specifies the maximum charge for a particular group of contacts. Equations (49), (50) 277 and (51) are in the standard form for a linear program and are solvable in polynomial ${ }_{278}$ time.

\section{Numerical Simulations}

The instantaneous response tells us how the amplitude of a system should change as a ${ }^{281}$ function of its state variables but did not take into account the dynamics of the system, ${ }^{282}$ such as the coupling which acts to resynchronise the oscillators and the effects of a finite ${ }^{283}$ number of oscillators - the latter leading to a breakdown in the underlying assumptions ${ }^{284}$ which lead to Equation (32). To better assess the real world performance of a particular 285 stimulation strategy we use the time-averaged response, which requires us to simulate 286 a system using equations (4), (27) and (26). 


\subsection{Simulated systems}

We define a system in terms of its electrode-population configuration, dynamics and intrinsic response to stimulation $Z(\theta)$. To construct a particular system we first randomly choose the coordinates of $S$ populations such that they lie within a box of length $L_{\text {box }}=10$. We then assign to each population an electrode, which we place $d_{\text {norm }}$ distance from the population. For a sufficiently large $L_{\text {box }}, d_{\text {norm }}$ can be used to characterise the system- a small $d_{\text {norm }}$ means the effects of stimulation are localised to a particular population and increasing $d_{\text {norm }}$ increasingly delocalises the effects of stimulation. For simplicity, we consider a system consisting of $S=4$ populations and $L=4$ electrodes. The analytical expressions for the response curves are for an infinite system, so we require that $N_{\sigma}$ is large. For each population, we choose the number of oscillators $N_{\sigma}=200$ to satisfy this and to remain computationally feasible. We also assume a coulombic system, where each electrode is able to simultaneously record and stimulate. In this case, the elements of $D$ are given by

$$
d_{l \sigma}=\frac{c w_{\sigma}}{\left|\mathbf{p}_{l}^{\prime}-\mathbf{P}_{\sigma}\right|}
$$

We denote the elements of matrix $\tilde{\mathbf{D}}$ as $\tilde{d}_{\sigma l}$, which can be related to $\mathbf{D}$ using the transpose

$$
\tilde{d}_{\sigma l}=\frac{d_{l \sigma}}{c w_{\sigma}}
$$

The dynamics of a system are determined by the parameters of the multipopulation Kuramoto model with an additional noise term

$$
d \theta_{\sigma n}=\left[\omega_{\sigma n}+\sum_{\sigma^{\prime}=1}^{S} w_{\sigma^{\prime}} k_{\sigma \sigma^{\prime}} \rho_{\sigma^{\prime}} \sin \left(\psi_{\sigma^{\prime}}-\theta_{\sigma n}\right)+V_{\sigma}(t) Z_{\sigma}\left(\theta_{\sigma n}\right)\right] d t+\tilde{\sigma} N(0,1) \sqrt{d t}
$$

To simplify our testing, we fix the basic parameters of (54) to those found from fitting ${ }^{289}$ to Patient 5. As previously mentioned, the natural frequencies $\left\{\omega_{\sigma n}\right\}$ are sampled from 290 a normal distribution. Such simulations represent a greater test for the robustness of 291 the predicted amplitude response due to stimulation (32), which assumes a Lorentzian ${ }^{292}$

distribution for $\left\{\omega_{\sigma n}\right\}$. 293

The $S \times S$ coupling constant matrix can be simplified by focussing only on the ${ }^{294}$ diagonal and off-diagonal components, which we denote by $k_{\text {diag }}$ and $k_{\text {offdiag }}$, respectively. $\quad 295$ We fix $k_{\text {offdiag }}=6$, so that $k_{\text {diag }}$ can be used to control the level of clustering for a ${ }^{296}$ particular configuration of oscillators- increasing $k_{\text {diag }}$ leading to increasingly multi- ${ }_{297}$ modal distributions of oscillators. The $\mathrm{nPRC} Z(\theta)$ was also chosen according to 298 parameters fitted to Patient 5, but we allow the zeroth harmonic $a_{0}$ to vary. 299

\subsection{Running the simulation 300}

To test each strategy we first create a system according to the set of parameters 301 $\left\{d_{\text {norm }}, k_{\text {diag }}, a_{0}\right\}$ then choose a stimulation strategy from CR, phase-locked (PL) and 302 
(a)
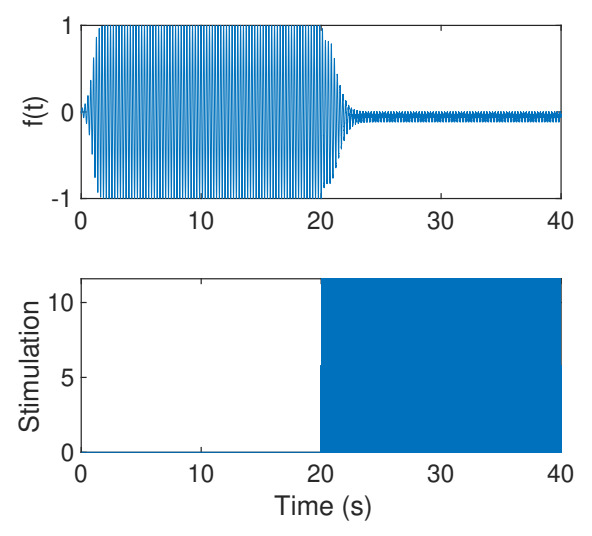

(b)

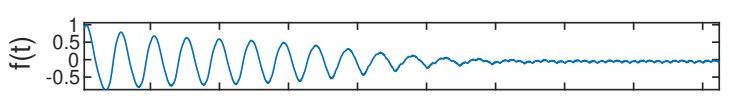

매매IIIIIIIIIIII

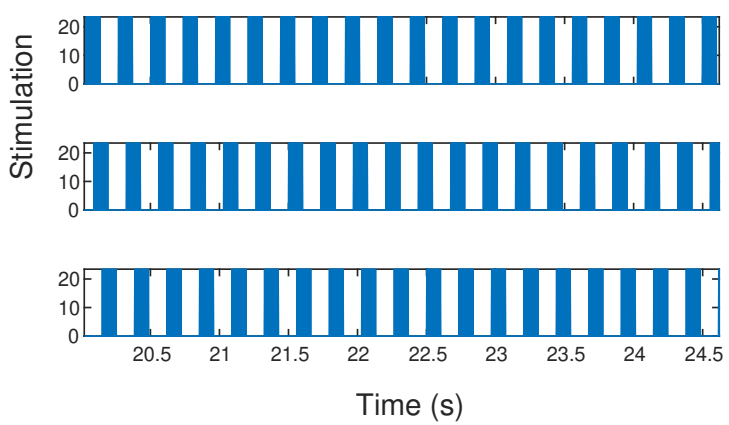

Figure 7. Output from numerical simulations showing the effects of Coordinated Reset (CR). Stimulation was turned on at $t=20$ seconds. The top panel of (a) shows the model output for a system simulated according to Equations (54) and (24). The bottom panel of (a) shows the stimulation delivered as a function of time, taken to be the average of the charges across the contacts. The bottom panel of (b) shows the stimulation across each contact, with the corresponding model output provided in the top panel.

ACR. Our implementation of PL stimulation is to use Equation (33), but to neglect all 303 the local terms, which is equivalent to setting $\rho_{\sigma}=0$.

We use the time-shifted variant of $\mathrm{CR}$ neuromodulation $[7,33]$ in our testing. For a given electrode, stimulation is delivered in bursts of HF pulse trains. The stimulation pattern is time-shifted across each electrode indexed by $l$ by

$$
\tau_{l}=\frac{\pi}{2 \bar{\omega}}(l-1)
$$

where $\bar{\omega}$ is the mean of the natural frequencies $(\simeq 4.2 \mathrm{~Hz})$. The number of bursts per 305 second, the burst frequency $f_{\text {burst }}$, was chosen to be equal to $\bar{\omega} / 2 \pi$ and the HF pulse 306 train frequency $f_{\text {train }}$ was chosen to be $130 \mathrm{~Hz}$. The width of each burst $t_{\text {burst }}$ was chosen 307 to be 0.1 seconds. Tass et al originally tested CR on a homogeneously coupled system 308 with $s_{\omega}=0$ [7]. We test our implementation and reproduce these results by constructing 309 a simple homogeneously coupled system according to the parameters of Patient 5 given 310 in Table 2, but with $\tilde{\sigma}=0, s_{\omega}=0$ and the parameters of $Z(\theta)$ scaled by a factor of ${ }_{311}$ 10. The simulation parameters were chosen according to Table 3 . The desynchronising 312 effects of CR neuromodulation on this system are shown in Figure 7, which reproduces 313 the results of Tass et al [7]. 314

The maximum charge for an electrode $q_{\max }^{\prime}$ is chosen so that, for a given system, 315 the maximum perturbation to a single oscillator is $d \theta_{\max }=0.2 \pi \mathrm{rad}$. For our testing, 316 


\begin{tabular}{|l|l|l|}
\hline Parameter & Value & Description \\
$\Delta t$ & & \\
$T$ & 0.04 & Integration time step \\
$L_{\text {box }}$ & 10 & Box length \\
$L$ & 4 & Number of electrodes \\
$S$ & 4 & Number of populations \\
$N_{\sigma}$ & 200 & Number of oscillators per population \\
$k_{\text {offdiag }}$ & 6 & Off-diagonal of coupling constant matrix \\
$d \theta_{\text {max }}$ & $0.2 \pi$ & Maximum angle moved per stimulation pulse \\
$n_{\text {trials }}$ & 24 & Number of trials \\
$f_{\text {max }}$ & 130 & Maximum frequency for PL stimulation \\
$f_{\text {burst }}$ & 4.2 & CR burst frequency \\
$f_{\text {train }}$ & 130 & CR HF train frequency \\
$t_{\text {burst }}$ & 0.1 & CR burst time \\
$\bar{\omega} / 2 \pi$ & 4.2 & Mean oscillator frequency \\
$s_{\omega} / 2 \pi$ & 0.7 & Standard deviation of oscillator frequency \\
\hline
\end{tabular}

Table 3. Summary of fixed parameters used in the simulations.

we do not constrain ACR using Equation (51), which leads to trivial optimal solutions 317 to the linear program (49) and (50), where the charge for the $l$ th contact is set to $q_{\max }^{\prime}$ if ${ }_{318}$ the $l$ th component of $\left(\tilde{\mathbf{D}}^{\top} \boldsymbol{\Gamma}\right)^{\top}$ is negative. ACR was tested at 3 maximum stimulation ${ }_{319}$ frequencies: $5 \mathrm{~Hz}, 50 \mathrm{~Hz}$ and $130 \mathrm{~Hz}$. The maximum stimulation frequency for PL was 320 fixed at $130 \mathrm{~Hz}$. Equation (54) was then integrated using Euler's method with a time ${ }_{321}$ step of $\Delta t=0.04$ seconds and simulated for $T=2$ seconds. The PL and ACR strategies ${ }_{322}$ were applied according to phases and amplitudes obtained directly from the simulation. ${ }_{323}$

During a simulation, a stimulation pulse is calculated as the average of the charges 324 $q^{\prime}(t)$ across the $L$ electrodes. Two quantities are calculated after each simulation: the ${ }_{325}$ time-averaged value of $\rho, \bar{\rho}$ and the total of all stimulation pulses $E$ delivered. The ${ }_{326}$ former is indicative of the efficacy of the strategy while the latter is related to the total ${ }_{327}$ energy consumption of a strategy, which can be used to gauge efficiency. For each set of ${ }^{328}$ parameters, the simulations are repeated over 24 trials, with a new electrode-population ${ }_{329}$ configuration being generated according to $d_{\text {norm }}$ for each trial. The parameters $d_{\text {norm }}{ }_{330}$ and $k_{\text {diag }}$ were chosen within the range $d_{\text {norm }} \in[0.1,6]$ and $k_{\text {diag }} \in[5,150]$. Example 331 output from these simulations, showing the effects of applying ACR, is provided in 332 Figure 8. When compared to Figure 7, it is clear that the stimulation pattern from ${ }_{333}$ ACR is significantly different from that produced by CR, with the latter pattern being ${ }_{334}$ simply time-shifted across electrodes. The stimulation pattern from ACR allows for the ${ }_{335}$ possibility that multiple electrodes may be stimulated simultaneously. A summary of 336 the parameters used in these simulations is provided in Tables 3 and 4 . 


\begin{tabular}{|l|l|l|l|l|}
\hline Parameter & Min & Max & $N_{\text {grid }}$ & Description \\
\hline$d_{\text {norm }}$ & 0.1 & 6 & 5 & Distance from population to electrode \\
$k_{\text {diag }}$ & 5 & 150 & 5 & $\begin{array}{l}\text { Diagonal of coupling constant matrix } \\
a_{0}\end{array}$ \\
\hline
\end{tabular}

Table 4. Summary of variable parameters used in the simulations. Each parameter was chosen in the range [Min,Max] using a uniform grid of spacing (Max-Min) $/ N_{\text {grid }}$.

(a)
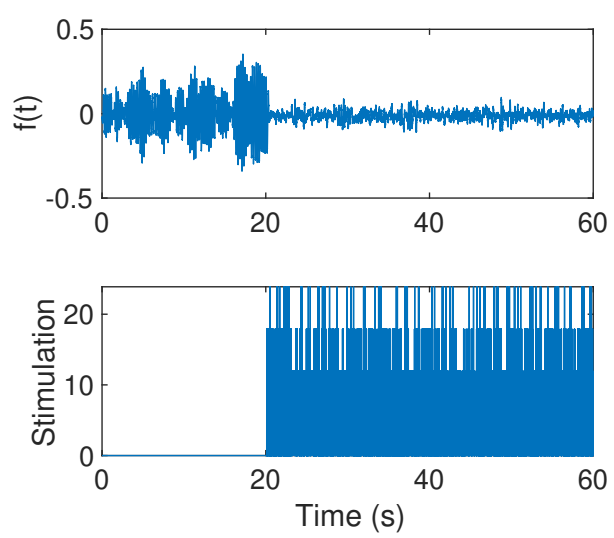

(b)
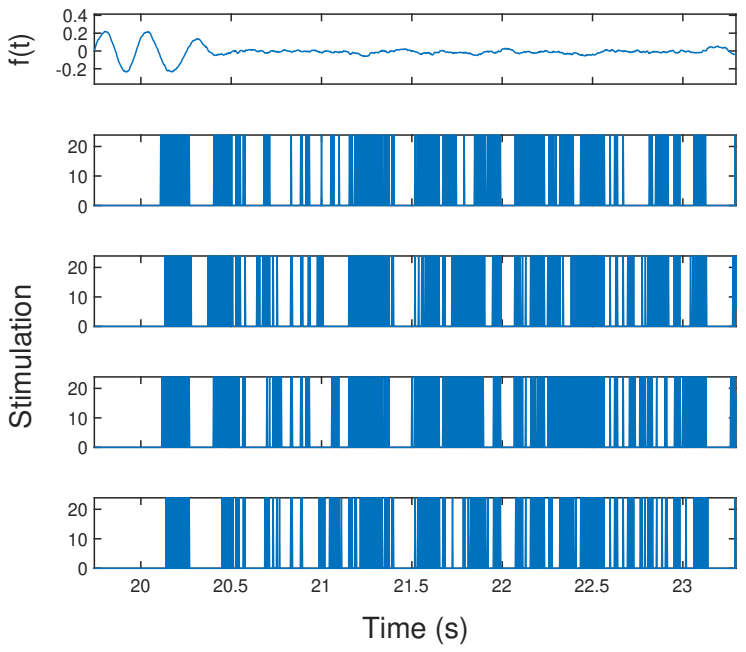

Figure 8. Output from numerical simulations showing the effects of Adaptive Coordinated Reset (ACR). Stimulation was turned on at $t=20$ seconds. The top panel of (a) shows the model output for a system simulated according to Equations (54) and (24). The bottom panel of (a) shows the stimulation delivered as a function of time, taken to be the average of the charges across the contacts. The bottom panel of (b) shows the stimulation across each contact, with the corresponding model output provided in the top panel.

\subsection{Results}

Figure 9 shows plots for the average amplitude $\bar{\rho}$, i.e. $\rho$ averaged over all trials and all 339 values of $d_{\text {norm }}$ for a particular value of $k_{\text {diag }}$ and zeroth harmonic $a_{0}$. ACR was tested 340 at maximum frequencies of $130 \mathrm{~Hz}, 50 \mathrm{~Hz}$ and $5 \mathrm{~Hz}$. The maximum frequency of PL ${ }_{341}$ was fixed at $130 \mathrm{~Hz}$. The rise in $\bar{\rho}$ shown between $k_{\text {diag }}=50$ and $k_{\text {diag }}=70$ is indicative ${ }_{342}$ of a bifurcation, which is typical in Kuramoto systems [26]. Significant improvements 343 with ACR over PL and CR are observed in simulations when stimulation is delivered 344 at higher frequencies and when $a_{0}$ is non-negligible. The utility of ACR over other 345 methods is also shown to be greatest when $k_{\text {diag }}$ is larger, which corresponds to larger ${ }_{346}$ local amplitudes $\rho_{\sigma}$ and increased clustering.

The efficacy of CR can also be seen to improve with systems with larger $a_{0}$. With ${ }_{348}$ 
(a)

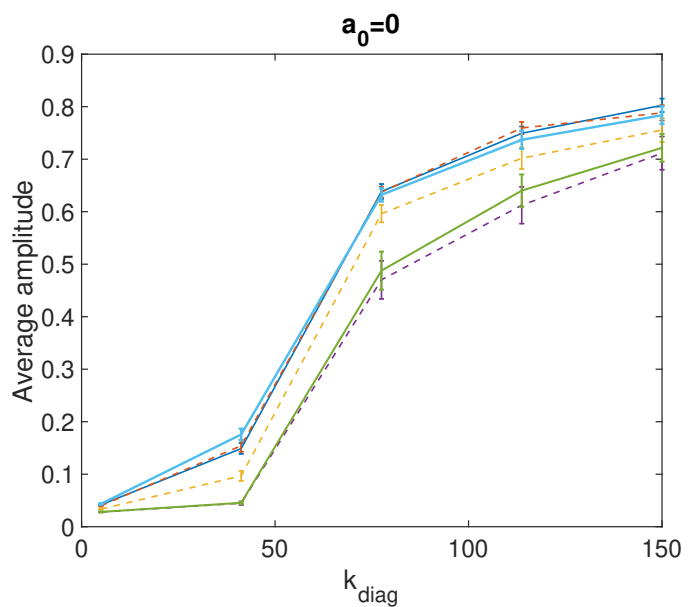

(c)

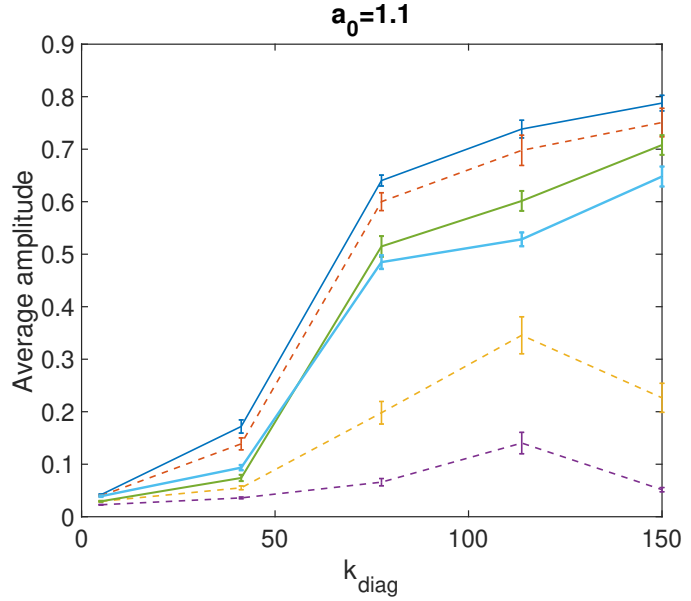

(b)

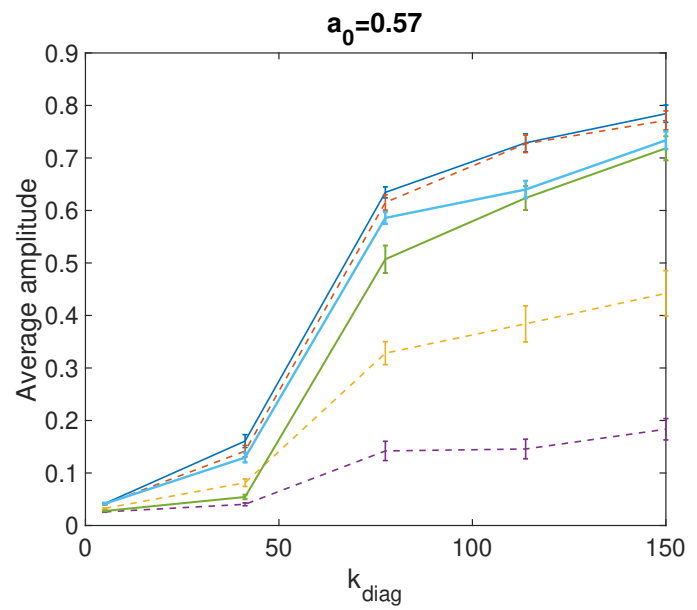

(d)

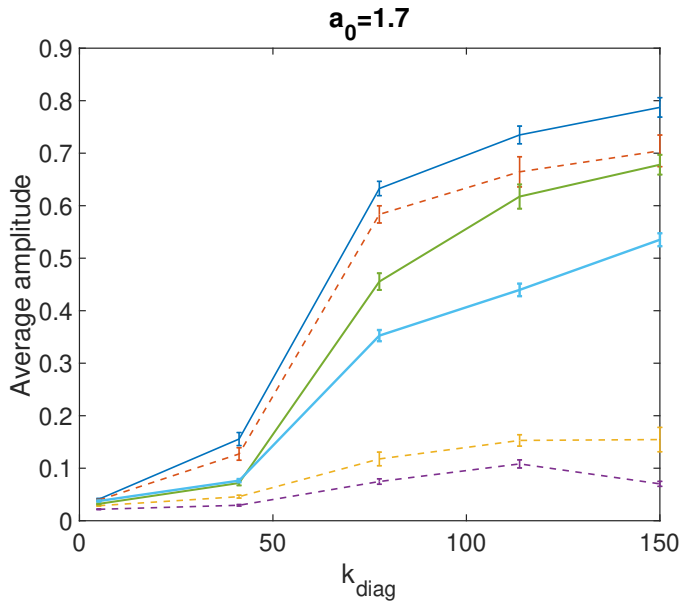

Figure 9. The average amplitude of a simulated Kuramoto system with a coupling constant $k_{\text {diag }}$ for different stimulation strategies: no stimulation (no stim), Adaptive Coordinated Reset (ACR), phase-locked (PL) and Coordinated Reset (CR). The maximum stimulation frequency used for $\mathrm{ACR}$ and $\mathrm{PL}$ is also given in the legend. Dashed lines are for the ACR method. Each sub plot shows a set of simulations performed with a particular zeroth harmonic of the nPRC $a_{0}$.

$a_{0}=0, \mathrm{CR}$ and no stimulation are shown to be equally effective. The results shown in ${ }_{349}$ Figure 9 are in contrast with those shown in Figure 7, indicating that the efficacy of CR 350 is sensitive to the parameters $\tilde{\sigma}$ and $s_{\omega}$ in addition to the scaling of $Z(\theta)$. 351

For systems where $a_{0} \simeq 0, \mathrm{PL}$ and $\mathrm{ACR}$ are found to be equally as effective, as 352 predicted. The efficacy of ACR at $130 \mathrm{~Hz}$ is greater than at other frequencies, but with ${ }_{353}$ more energy usage than PL and similar energy usage to CR, as shown in Figure 10. ACR ${ }_{354}$ at $50 \mathrm{~Hz}$ is found to have good efficacy for $a_{0}>0$ but with significantly less energy usage 355 than PL and CR. ACR with low frequency stimulation at $5 \mathrm{~Hz}$ (or approximately the 356 tremor frequency) is predicted to have little to no effect on all the systems tested. ${ }_{357}$

As previously mentioned, we expect the utility of ACR to be greatest for those 358 systems described by type I nPRCs as the amplitude response curve then depends 359 
-I ACR $(5 \mathrm{~Hz})-\mathrm{ACR}(50 \mathrm{~Hz})-\mathrm{F}$ ACR $(130 \mathrm{~Hz})-\mathrm{PL}(130 \mathrm{~Hz}) \mp \mathrm{CR}$

(a)

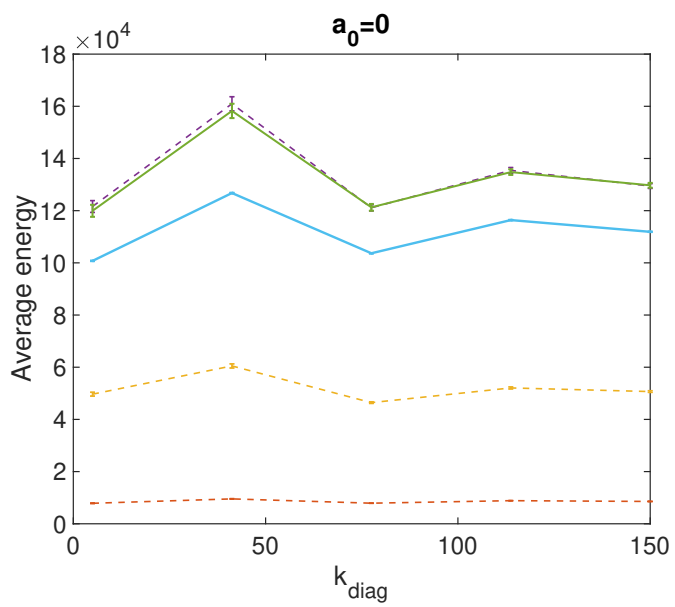

(c)

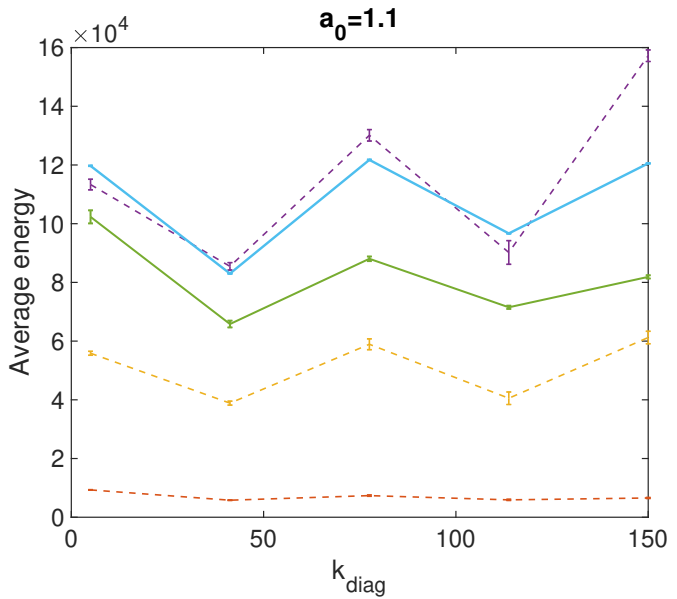

(b)

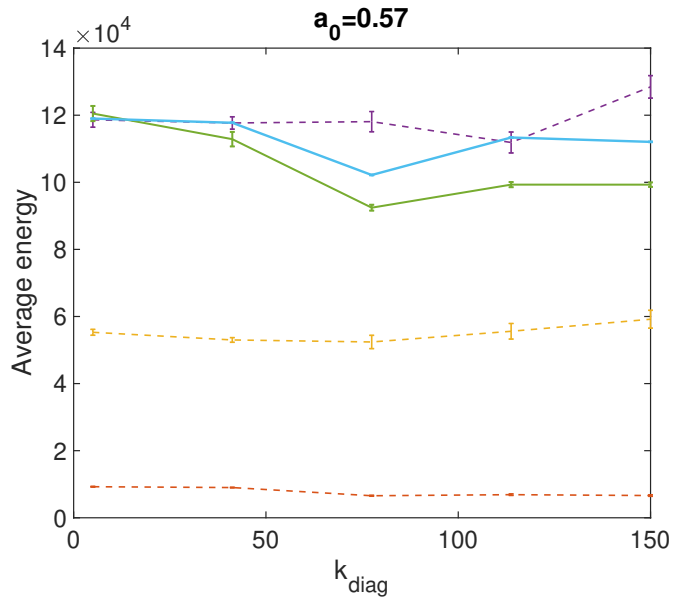

(d)

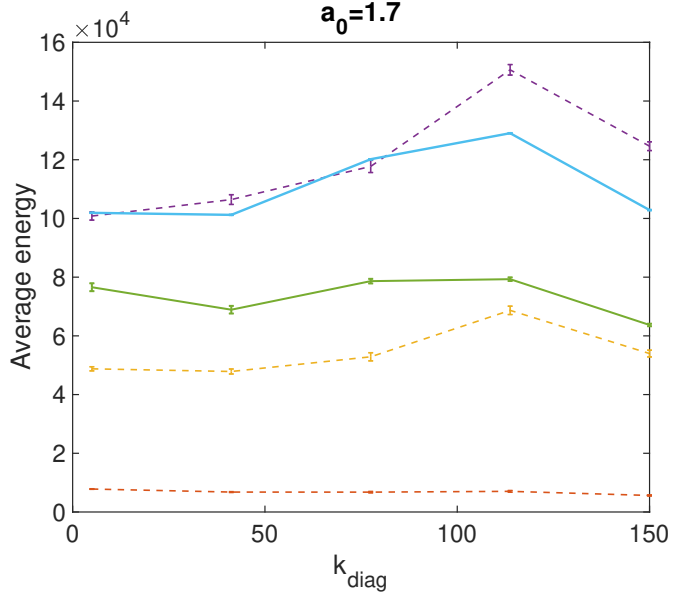

Figure 10. The average energy used by: no stimulation (no stim), Adaptive Coordinated Reset (ACR), phase-locked (PL) and Coordinated Reset (CR) stimulation strategies on a simulated Kuramoto system with a coupling constant $k_{\text {diag. }}$. The maximum stimulation frequency used for ACR and PL is also given in the legend. Dashed lines are for the ACR method. Each sub plot shows a set of simulations performed with a particular zeroth harmonic of the nPRC $a_{0}$.

explicitly on non-negligible terms involving population quantities. Equation (32) shows 360 that when $a_{0}=0$, the terms involving population quantities depend on second harmonics 361 $2 \psi_{\sigma}$. These terms carry a factor of $\rho_{\sigma}^{2}$ and hence are likely to be negligible, except when ${ }^{362}$ $\rho_{\sigma}$ is reasonably large. To investigate these effects, we simulate systems with $a_{0}=0{ }^{363}$ and use a larger stimulation intensity $q_{\max }^{\prime}$. By comparing ACR to PL, we can then 364 ascertain the impact of these second harmonic terms. A comparison of the efficacy of 365 ACR with PL and CR, is shown in Figure 11 for different stimulation amplitudes $q_{\max }^{\prime}{ }^{366}$ and with $a_{0}=0$. Increasing stimulation amplitude can be seen to improve the efficacy 367 of all the strategies tested, but is most noticeable for ACR and PL. Higher stimulation ${ }_{368}$ amplitudes also seem to be particularly beneficial for those systems with larger $k_{\text {diag. }}{ }_{369}$ ACR can be seen to perform better than CR in all cases. Figure 11 shows the ACR 370 
(a)

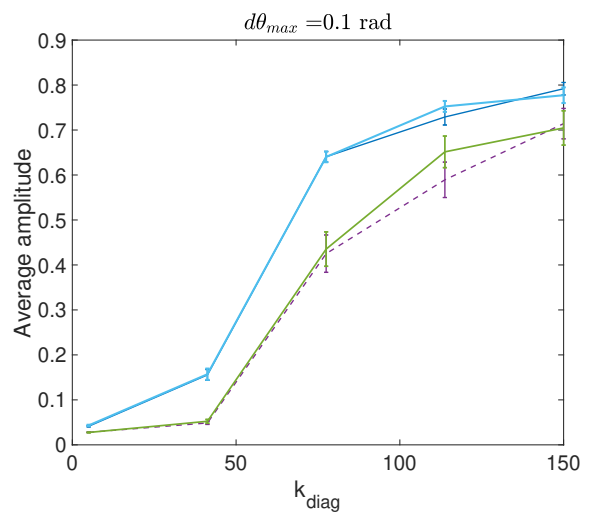

(c)

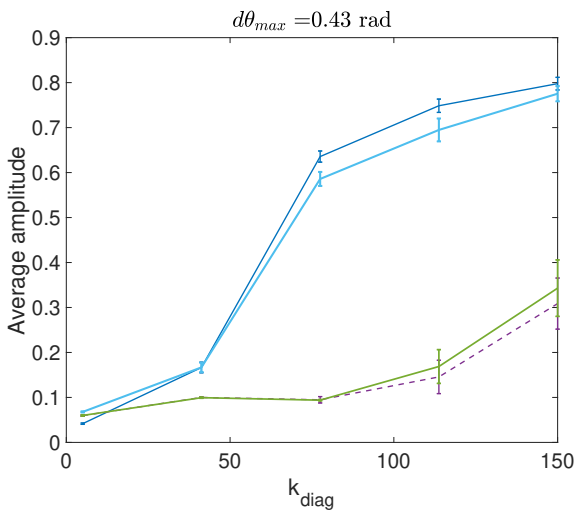

(b)

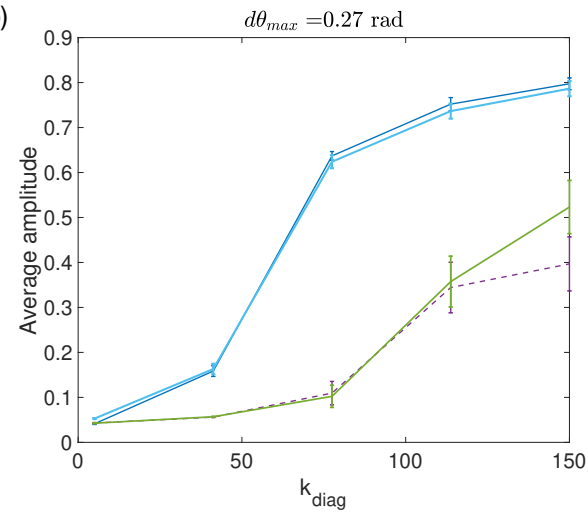

(d)

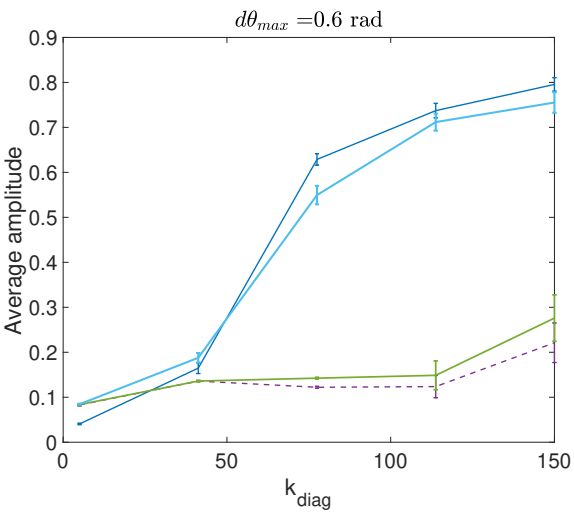

Figure 11. The average amplitude of a simulated Kuramoto system with a coupling constant $k_{\text {diag }}$ for different stimulation strategies: no stimulation (no stim), Adaptive Coordinated Reset (ACR), phase-locked (PL) and Coordinated Reset (CR). During these simulations, the zeroth harmonic $a_{0}$ of the $\mathrm{nPRC} Z(\theta)$ was fixed to zero. Increasing $d \theta_{\max }$ leading to larger stimulation amplitudes $q_{\max }^{\prime}$ shows the effect of the second harmonic term in the amplitude response given by Equation (32).

method to be consistently more effective than PL at higher $k_{\text {diag }}$, although the difference ${ }_{371}$ is marginal. This is indicative of the aforementioned effects of second harmonic terms in 372 (32). Taken altogether, we predict the efficacy of ACR to be similar to PL stimulation ${ }_{373}$ for those systems where $a_{0} \simeq 0$.

\section{Discussion}

In this work we propose ACR as a method for DBS using multiple contacts. Unlike CR, ${ }^{376}$ the method is closed-loop and uses information about the system to determine when to 377 apply stimulation. Using numerical simulation, we show that in many cases, substantial 378 improvements to the efficacy can be achieved with the method. The mathematical 379 description of ACR also predicts that the effectiveness of multi-contact stimulation is 380 largely dependent on the form of the $\mathrm{nPRC}$ and in particular on the zeroth harmonic 381 $a_{0}$, which is related to whether it is type I or type II. We predict that for type II ${ }_{382}$ systems, where $\left|a_{0}\right|$ is small, stimulation on the basis of local quantities is unlikely to 383 
be beneficial. We also show that the dependency of the amplitude response on the local 384 quantities of population $\sigma$ becomes less at increasingly lower local amplitudes $\rho_{\sigma}$ but 385 that the effects of stimulation are, in general, explicitly dependent on the state of the ${ }_{386}$ system and that providing stimulation without knowledge of this state is likely to be ${ }_{387}$ suboptimal. Following from this, it is worth discussing the feasibility of resolving this 388 state in practice.

\subsection{Practicalities of Using ICA}

The ACR method assumes an underlying system of phase oscillators, which can be divided into small populations with the distribution of oscillators in each population satisfying the ansatz of Ott and Antonsen [26]. Equation (44) links the state to measurable quantities from the electrode and is of the form modelled by ICA [32]. The goal of ICA here is to resolve the $S$ population quantities from $L$ electrode measurements. Variations of the ICA problem exist depending on whether $S<L$ (the overdetermined case), $S>L$ (the underdetermined case) and $S=L$ (the determined case). The determined case is perhaps the most common and more easily solved, since the mixing matrix $D$ is invertible. However, we do not know the value of $S$ a priori and therefore cannot know which ICA method is best suited. If we assume the case of $S=L$, then ICA will always resolve exactly $L$ components. With this assumption, increasing the number of electrodes in a system has a definite purpose: it increases our potential to resolve the internal state. Assuming a larger number of populations also increases the validity of the small region approximation presented in Equation (38) and thus the accuracy of ACR. It may also be possible to obtain good approximations to the state by using $L<S$ electrodes, since in some cases the weights $w_{\sigma}$ may be small for some populations and can hence be neglected. This together with the statistical nature of ICA, errors due to applying various signal processing techniques and noise within measurement would inevitably lead to some uncertainty when determining the population quantities. In addition to this, the amplitude response (32) is also dependent on the harmonics of $Z(\theta)$, which also need to be determined. Electrodes which record the population activity are also susceptible to recording the stimulation pulses themselves. methods that rely on the real-time measurement of phases and amplitudes. Addressing the effects of stimulation artefacts is beyond the scope of this work, but we expect that significant suppression of the stimulation artefact would be required for ACR to be effective. This suppression may come as a byproduct of using ICA, which has been found by others $[34,35]$. Alternatively, by recording through two contacts adjacent to a single stimulating contact, the properties of differential amplifiers can be used to suppress the stimulation artefact [36]. In this paper we have considered perturbations to neural populations using electrodes, but in principle, our theories should also be valid for other types of perturbation, such as optogenetic, where light pulses are used to ${ }_{422}$ perturb genetically modified neurons. This approach would eliminate the stimulation ${ }_{423}$ 
artefact and thus likely improve the real-world performance of ACR. In summary, the ${ }_{424}$ effectiveness of ACR in practice is likely to be dependent on both the ability of the ${ }_{425}$ model to capture the underlying dynamics of the system and our ability to resolve the ${ }_{426}$ state and parameters of the system. It is worth mentioning that the latter does not ${ }_{427}$ factor into the results presented in Section 5 since both the state and the parameters ${ }_{428}$ were taken directly from the simulation. Therefore the results we present for ACR (and ${ }_{429}$ PL) can be taken as an upper bound on performance.

As a preliminary study, the simulations presented in this work provide a broad ${ }_{432}$ understanding of the potential efficacy and efficiency of ACR but there is scope for future ${ }^{433}$ work. Firstly, we could test the simulation on a greater variety of systems, changing both ${ }_{434}$ the distribution of natural frequencies, the noise parameter and the harmonics of $Z(\theta)$. ${ }^{435}$ Secondly, we could investigate how the constraints of the linear program (51) affect ${ }_{436}$ both the efficacy and efficiency of ACR. In addition to these, there is also considerable ${ }_{437}$ scope for understanding both the potential and effective real world performance of ACR, ${ }^{438}$ which could involve investigating the performance of ACR with the harmonics of $Z(\theta) \quad{ }^{439}$ estimated through machine learning, using the state variables obtained through ICA ${ }_{440}$ and finally testing ACR on patients.

\section{Appendix}

\section{A. Model Fitting}

\section{A.1. Feature Selection}

In Section 3 we described how the similarity between two time series can be quantified using features extracted from the data. We define here a feature to be some transformation of the time series $F(t)$ into a new function $y(\zeta)$, where $\zeta$ is in a new domain. For example, the PSD can be obtained by applying the Fourier transformation to the time series with $\zeta$ being the frequency in this case. We can then characterise a time series using a set of features. This set, though arbitrary, should be chosen so as to reproduce important properties of the data. For a set of $N_{c}$ features, we can construct a cost function $C(\mathbf{X})$ for a vector of parameters $\mathbf{X}$ to be used in a local optimisation

$$
C(\mathbf{X})=\frac{1}{N_{c}} \sum_{j=1}^{N_{c}}\left(\frac{\left\|\mathbf{y}_{j}-\mathbf{y}_{j}^{\text {model }}(\mathbf{X})\right\|}{\left\|\mathbf{y}_{j}-\overline{\mathbf{y}}_{j}\right\|}\right)^{2},
$$

where $\mathbf{y}$ indicates a vector over the domain $\zeta$. Here $\mathbf{y}$ and $\mathbf{y}^{\text {model }}$ are the features ${ }_{445}$ from the experimental and simulated data, respectively. The mean of an experimental ${ }_{446}$ feature is denoted by $\overline{\mathbf{y}}$. Each term in the summation is simply $\left(1-R^{2}\right)$, where $R^{2}$ is the ${ }^{447}$ standard coefficient of determination. Qualitatively, Equation (A.1) is simply the mean ${ }_{448}$ of $\left(1-R^{2}\right)$ across all the features. Equation (A.1) quantifies the similarity between ${ }_{449}$ 
(a)
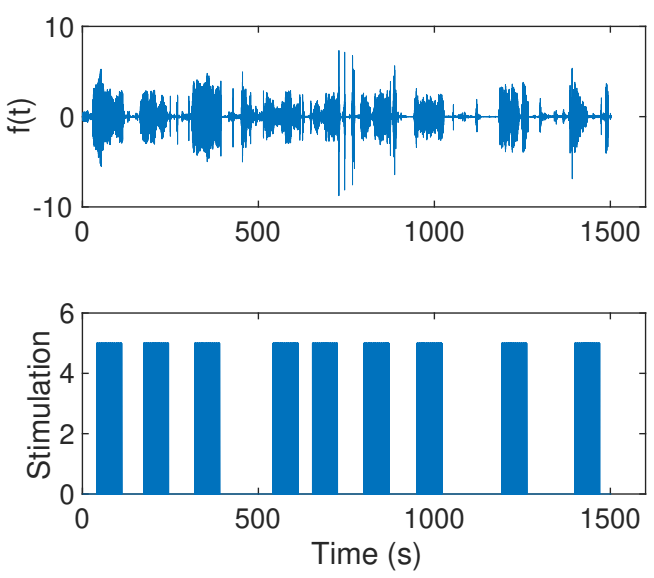

(b)
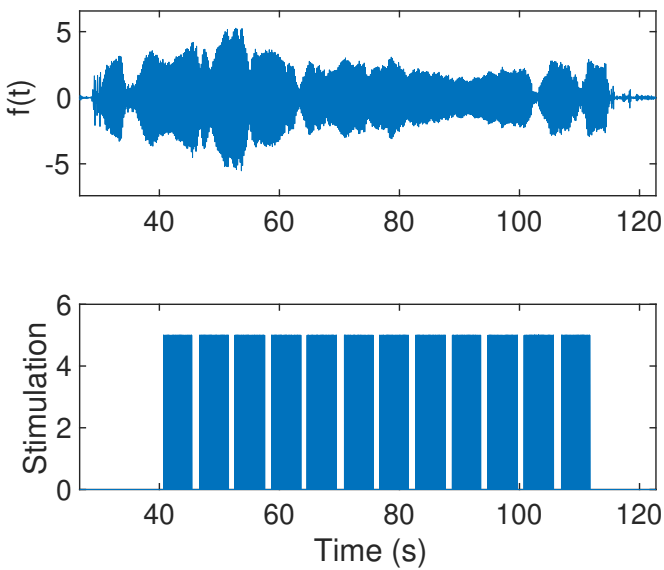

Figure A1. Experimental data for Patient 1 from the study of Cagnan et al [12]. Tremor oscillations are shown in the top panels. The bottom panels shows the stimulation triggers. (a) shows the entirety of the dataset consisting of stimulation provided over 9 trials. (b) shows a single trial which consists of 5 seconds of phaselocked stimulation over 12 phases.

features obtained from simulated and experimental data. It can be seen that $C(\mathbf{X})=0 \quad{ }_{450}$ implies both sets of features are equal. When $C(\mathbf{X})=1$, the fit of the model is no ${ }_{451}$ better than the mean $\overline{\mathbf{y}}$. As previously mentioned in Section 3, the features reflecting ${ }_{452}$ the dynamics of the oscillations are chosen to be: the PSD, the PDF for the amplitude ${ }^{453}$ and the PSD of the envelope amplitude. We also use the averaged PRC as an additional ${ }^{454}$ feature to characterise the response of a particular patient.

\section{A.2. Experimental data}

Cagnan et al [12] studied phase-locked DBS delivered according to the tremor in ET patients. Data was collected from 6 ET patients and 3 dystonic tremor patients. All 457 patients gave their informed consent to take part in the study, which was approved by the local ethics committee in accordance with the Declaration of Helsinki. The data from this study can be obtained through an online repository [29].

Duchet et al [28] defined a criterion for assessing significance in the averaged ARCs and PRCs from the study of Cagnan et al. In their study, a patient is considered to have a significant response if both the ARC and PRC are found to be significant either according to an ANOVA test or cosine model F-test. Using this, they deemed 3 out of the 6 ET patients to have a significant response curve. We restrict our analysis to these 3 patients, who we shall refer to as patients 1,5 and 6, as in the original study. The tremor data was filtered using a non-causal Butterworth filter of order 2 with cut-off frequencies at $\pm 2 \mathrm{~Hz}$ around the tremor frequency. Stimulation was delivered over a set of trials (typically 9), with each trial consisting of 12 blocks of 5 second phase-locked 458 459 


\begin{tabular}{|l|l|l|}
\hline Parameter & Value & Description \\
\hline & & \\
$T t$ & 0.002 & Integration time step \\
$T_{i b}$ & 5 & Stimulation time \\
$T_{i t}$ & 1 & Inter-block time \\
$N_{\sigma}$ & 60 & Inter-trial time \\
$n_{\text {trials }}$ & 54 & Number of oscillators \\
\hline
\end{tabular}

Table A1. Parameters used when simulating the Kuramoto model for global optimisation.

stimulation was also separated by a 1 second interblock of no stimulation. The envelope ${ }_{472}$ amplitude and instantaneous phase were calculated using the Hilbert transform. As an ${ }_{473}$ example, the data for Patient 1 is shown in Figure A1. From this, the characteristics we ${ }_{474}$ identify as being desirable for our model to reproduce are: the frequency spectrum of ${ }_{475}$ the data, the bursts of oscillations and the sustained periods of low envelope amplitude. ${ }_{476}$ In addition to this we would also like the model to reproduce a given patient's response ${ }_{477}$ to stimulation, as characterised by the averaged PRC.

\section{A.3. Simulated data}

For the $m$ th local optimisation step, we simulate the Kuramoto model (18) using a ${ }_{480}$ parameter set $\mathbf{X}_{m}$ and obtain the feature set described in Section A.1. The stochasticity ${ }_{481}$ of the model naturally leads to variation in the features for a particular optimisation ${ }_{482}$ step. To stabilise this variation we average the features over $n_{\text {trials }}=54$ trials. The ${ }^{483}$ simulation was configured to reproduce the methodology of Cagnan et al [12], namely ${ }^{484}$ that stimulation was delivered in blocks of trials, as described in Section A.2. A summary ${ }^{485}$ of the parameters used in the simulations is provided in Table A1.

Calculation of the cost function $C(\mathbf{X})$ requires us to obtain the feature set for ${ }_{487}$ each instance of the simulation. The averaged PRC can be obtained according to the ${ }_{488}$ methods described in our previous paper [14] and elsewhere [12, 28]. The method is ${ }_{489}$ suitable for both experimental and simulated data, but can be computationally costly ${ }_{490}$ and generally unsuitable in an optimisation setting. To ensure computational feasibility, 491 we use an approximation for the averaged PRC for part of the optimisation. A suitable ${ }^{492}$ approximation should be computationally cheap, stable and reasonably accurate. The ${ }_{493}$ requirement of stability precludes the use of the analytical expressions for the PRC ${ }_{494}$ (17), which are derived on the basis of an infinite system of oscillators satisfying ${ }_{495}$ the ansatz of Ott and Antonsen [26]. Situations affecting stability which may arise ${ }^{496}$ during optimisation include large values of the noise parameter $\tilde{\sigma}$, which may lead to a ${ }_{497}$ breakdown in the assumptions underlying (17). This motivates the need for alternative ${ }_{498}$ method, which we present here.

Assuming an infinite system of oscillators, the order parameter $r$ can be written in 
integral form

$$
r=\int_{0}^{2 \pi} F(\theta, t) e^{i \theta} d \theta
$$

where $F(\theta, t)$ is the PDF for the oscillators. Differentiating with respect to time gives

$$
\frac{d r}{d t}=\int_{0}^{2 \pi}\left(\frac{\partial F(\theta, t)}{\partial t}\right) e^{i \theta} d \theta
$$

Using the stimulation part of (18), the continuity equation for $F(\theta, t)$ due only to stimulation can be written as

$$
\frac{\partial F(\theta, t)}{\partial t}=-V \frac{\partial}{\partial \theta}[Z(\theta) F(\theta, t)]
$$

Inserting (A.4) into (A.3) gives

$$
\frac{d r_{s t i m}}{d t}=-V \int_{0}^{2 \pi} \frac{\partial}{\partial \theta}[Z(\theta) F(\theta, t)] e^{i \theta} d \theta .
$$

Using the polar form for $\frac{d r_{s t i m}}{d t}$ in (A.5) gives

$$
\frac{d \rho_{\mathrm{stim}}}{d t}+i \rho \frac{d \psi_{\mathrm{stim}}}{d t}=-V \int_{0}^{2 \pi} \frac{\partial}{\partial \theta}[Z(\theta) F(\theta, t)] e^{i(\theta-\psi)} d \theta .
$$

Expressions for the instantaneous ARC and PRC can be found by equating the real and complex parts of (A.6), respectively, leading to

$$
\frac{d \rho_{\text {stim }}}{d t}=-V \int_{0}^{2 \pi} \frac{\partial}{\partial \theta}[Z(\theta) F(\theta, t)] \cos (\theta-\psi) d \theta
$$

and

$$
\frac{d \psi_{\text {stim }}}{d t}=-\frac{V}{\rho} \int_{0}^{2 \pi} \frac{\partial}{\partial \theta}[Z(\theta) F(\theta, t)] \sin (\theta-\psi) d \theta .
$$

The averaged PRC can be expressed using a summation over the time points of stimulation $\left\{t_{m}\right\}$

$$
\left\langle\frac{d \psi_{\text {stim }}}{d t}\right\rangle=-\frac{V}{N_{\psi}} \sum_{m=1}^{N_{\psi}} \frac{1}{\rho\left(t_{m}\right)} \int_{0}^{2 \pi} \frac{\partial}{\partial \theta}\left[Z(\theta) F\left(\theta, t_{m}\right)\right] \sin (\theta-\psi) d \theta
$$

If we group these time points according to the phase $\psi$, with $N_{\psi}$ points for the phase $\psi$, then we can express the averaged response in terms of the PDFs conditioned on $\psi$

$$
\left\langle\frac{d \psi_{\text {stim }}}{d t}\right\rangle=-V\left[\int_{0}^{1} \frac{G(\rho \mid \psi)}{\rho} d \rho\right] \int_{0}^{2 \pi} \frac{\partial}{\partial \theta}[Z(\theta) F(\theta \mid \psi)] \sin (\theta-\psi) d \theta .
$$

Equation (A.10) represents a computationally cheap way of estimating the averaged 500 PRC since the PDF for the amplitude conditioned on the phase $G(\rho \mid \psi)$ and the PDF ${ }_{501}$ for the oscillators $F(\theta \mid \psi)$ conditioned on the phase can be easily accumulated during 502 simulation. 
For each instance of the model output, we calculate the vector of features $\left\{\mathbf{y}_{j}^{\text {model }}(\mathbf{X})\right\}$ and their similarity with the experimental feature set $\left\{\mathbf{y}_{j}\right\}$ measured by $C(\mathbf{X})$. Since ${ }_{506}$ Equation (18) is a stochastic differential equation, the features $\left\{\mathbf{y}_{j}^{\text {model }}(\mathbf{X})\right\}$ are averaged ${ }_{507}$ over a certain number of trials. The global minimum of the cost function $C(\mathbf{X}){ }_{508}$ corresponds to the set of optimisable parameters which best reproduces the experimental ${ }_{509}$ features. Starting at a configuration $\mathbf{X}_{0}$, we can reach a local minimum of $C(\mathbf{X})$ using 510 local optimisation. By repeating this process using many randomly generated starting 511 configurations, a best fit can be obtained by taking the smallest local minimum. To ${ }_{512}$ obtain an initial configuration $\mathbf{X}_{0}$, we choose a value for each optimisable parameter ${ }_{513}$ by randomly sampling from a bounded uniform distribution. The bounds for each ${ }_{514}$ optimisable parameter are given in Table A2. Optimisation of the cost function $C(\mathbf{X}){ }_{515}$ was performed using custom written code in MATLAB. The local optimisation was 516 performed using the fminsearch function which uses the derivative-free Nelder-Mead simplex method of Lagarias et al [37].

Simultaneous optimisation of the Kuramoto parameters, together with those of oscillation dynamics and the averaged PRC. In order to be computationally feasible, we 521 performed the optimisation in stages. The principle here is to use a cheaper calculation 522 to push the local optimisation towards a local minimum and then gradually refine the optimisation using a higher quality calculation. First, we performed the optimisation without stimulation, only fitting to those features representing the dynamics. We then used the parameters from our best fit in a second optimisation, using the features representing the dynamics and the theoretical approximation to the averaged PRC, for computational efficiency. Finally, the parameters from this best fit were used in a final optimisation, where the averaged PRC feature was instead calculated using the experimental methodology.

The best fits found through optimisation are shown in Figures 1 (for the dynamics) and 2 (for the response). Instances of output for the fitted models are shown in Figure 2 together with experimental data included for comparison. The parameters found through optimisation are provided in Table 2.

\section{B. Implications for Single Contact DBS}

In this subsection we will review our results in the context of single contact DBS. Specifically, we want to understand the feasibility of a closed-loop DBS strategy which uses a feedback signal from a single contact. In the case of a single electrode contact, the voltage can be expressed as a summation over population activities using Equation (44)

$$
v_{l}^{\prime}(t)=\sum_{\sigma=1}^{S} d_{l \sigma} \rho_{\sigma} \cos \left(\psi_{\sigma}\right) .
$$




\begin{tabular}{|l|l|l|}
\hline Parameter & Min & Max \\
\hline & & \\
$k$ & 0 & 6 \\
$\tilde{\sigma}$ & 0 & 4 \\
$\bar{\omega} / 2 \pi$ & 4.5 & 5.5 \\
$s_{\omega} / 2 \pi$ & 0.5 & 1 \\
$a_{0}$ & -0.1 & 0.1 \\
$a_{1}$ & -0.09 & 0.09 \\
$b_{1}$ & -0.09 & 0.09 \\
$V$ & 0 & 0.6 \\
\hline
\end{tabular}

Table A2. Bounds for the optimisable parameters used in generating random initial configurations. An initial configuration is generated by randomly sampling from a uniform distribution for each parameter within the bounds.

Comparing this with the expression for the global signal (24) and (25) (with $c=1$ for simplicity) we can immediately see a correspondence between the matrix elements $\left\{d_{l \sigma}\right\}$ and the population weights $\left\{w_{\sigma}\right\}$. The matrix elements encode the electrostatic properties of the medium and the electrode-population geometry. In theory, therefore, positioning the electrode has the effect of changing the matrix elements in the expansion given by Equation (B.1). An expression for the amplitude and phase of $v_{l}^{\prime}(t)$ can be obtained using the analytic signal (1), namely

$$
\mathrm{P} e^{i \Psi}=v_{l}^{\prime}(t)+i \hat{H}\left[v_{l}^{\prime}(t)\right]
$$

Then, inserting (B.1) into (B.2) and using the approximation (8) leads to

$$
\mathrm{P} e^{i \Psi}=\sum_{\sigma=1}^{S} d_{l \sigma} \rho_{\sigma} e^{i \psi_{\sigma}}
$$

which has an identical form to (27). If the electrode is positioned such that the matrix elements coincide exactly with the population weights, although unlikely in general, then the amplitude $\mathrm{P}(t)$ would equal the synchrony $\rho$. In general, the electrode should be positioned so that $\mathrm{P}(t)$ is highly correlated to the symptom severity and hence $\rho$. Using (B.3), the derivation of the amplitude response due to stimulation can then proceed exactly as before, leading to an identical expression to (32) except with the population weights replaced with the matrix elements. Explicitly,

$$
\frac{d \mathrm{P}_{\text {stim }}}{d t} \simeq \frac{1}{2} \sum_{\sigma=1}^{S} d_{l \sigma} V_{\sigma}(t)\left\{\left[a_{1} \sin (\Psi)-b_{1} \cos (\Psi)\right]-\rho_{\sigma} a_{0} \sin \left(\psi_{\sigma}-\Psi\right)\right\} .
$$

Since, by definition, $\mathrm{P}(t)$ should be correlated to symptom severity, it follows that ${ }_{536}$ Equation (B.4) can be used in a closed-loop DBS strategy. From this we also conclude ${ }_{537}$ that the effectiveness of single contact closed-loop DBS should also be dependent on $\left|a_{0}\right|$. ${ }_{538}$ 
In the cases where $\left|a_{0}\right|$ is non-negligible, knowledge of the population quantities $\rho_{\sigma}$ and ${ }_{539}$ $\psi_{\sigma}$ would be required for an effective closed-loop strategy. Therefore, by estimating $\left|a_{0}\right|{ }_{540}$ for a particular system, we can go some way towards predicting the likely effectiveness ${ }^{541}$ of single contact closed-loop DBS.

\section{References}

[1] Thenganatt MA, Jankovic J. The relationship between essential tremor and Parkinson's disease. Parkinsonism \& related disorders. 2016;22:S162-S165.

2] Yousif N, Mace M, Pavese N, Borisyuk R, Nandi D, Bain P. A network model of local field potential activity in essential tremor and the impact of deep brain stimulation. PLoS computational biology. 2017;13(1):e1005326.

[3] Elble RJ. What is essential tremor? Current neurology and neuroscience reports. 2013;13(6):353.

[4] Little S, Pogosyan A, Neal S, Zavala B, Zrinzo L, Hariz M, et al. Adaptive deep brain stimulation in advanced Parkinson disease. Annals of neurology. 2013;74(3):449-457.

[5] Hua S, Lenz F, Zirh T, Reich S, Dougherty PM. Thalamic neuronal activity correlated with essential tremor. Journal of Neurology, Neurosurgery \& Psychiatry. 1998;64(2):273-276.

[6] Koller W, Pahwa R, Busenbark K, Hubble J, Wilkinson S, Lang A, et al. High-frequency unilateral thalamic stimulation in the treatment of essential and parkinsonian tremor. Annals of Neurology: Official Journal of the American Neurological Association and the Child Neurology Society. 1997;42(3):292-299.

[7] Tass PA. A model of desynchronizing deep brain stimulation with a demand-controlled coordinated reset of neural subpopulations. Biological cybernetics. 2003;89(2):81-88.

[8] Tass PA, Qin L, Hauptmann C, Dovero S, Bezard E, Boraud T, et al. Coordinated reset has sustained aftereffects in Parkinsonian monkeys. Annals of neurology. 2012;72(5):816-820.

[9] Popovych OV, Tass PA. Desynchronizing electrical and sensory coordinated reset neuromodulation. Frontiers in human neuroscience. 2012;6:58.

[10] Kubota S, Rubin JE. Numerical optimization of coordinated reset stimulation for desynchronizing neuronal network dynamics. Journal of computational neuroscience. 2018;45(1):45-58.

[11] Peles O, Werner-Reiss U, Bergman H, Israel Z, Vaadia E. Phase-Specific Microstimulation Differentially Modulates Beta Oscillations and Affects Behavior. Cell Reports. 2020;30(8):25552566 .

[12] Cagnan H, Pedrosa D, Little S, Pogosyan A, Cheeran B, Aziz T, et al. Stimulating at the right time: phase-specific deep brain stimulation. Brain. 2016;140(1):132-145.

[13] Grado LL, Johnson MD, Netoff TI. Bayesian adaptive dual control of deep brain stimulation in a computational model of Parkinson's disease. PLOS Computational Biology. 2018;14(12):e1006606.

[14] Weerasinghe G, Duchet B, Cagnan H, Brown P, Bick C, Bogacz R. Predicting the effects of 574 deep brain stimulation using a reduced coupled oscillator model. PLoS computational biology. 2019;15(8):e1006575.

[15] Brown E, Moehlis J, Holmes P. On the phase reduction and response dynamics of neural oscillator populations. Neural computation. 2004;16(4):673-715.

[16] Hansel D, Mato G, Meunier C. Synchrony in excitatory neural networks. Neural computation. 1995; $7(2): 307-337$.

[17] Schultheiss NW, Edgerton JR, Jaeger D. Phase response curve analysis of a full morphological globus pallidus neuron model reveals distinct perisomatic and dendritic modes of synaptic integration. Journal of Neuroscience. 2010;30(7):2767-2782.

[18] Cagnan H, Brittain JS, Little S, Foltynie T, Limousin P, Zrinzo L, et al. Phase dependent modulation of tremor amplitude in essential tremor through thalamic stimulation. Brain. 
[19] Holt AB, Kormann E, Gulberti A, Pötter-Nerger M, McNamara CG, Cagnan H, et al. 587 Phase-dependent suppression of beta oscillations in Parkinson's disease patients. Journal of 588 Neuroscience. 2019;39(6):1119-1134.

[20] Raethjen J, Deuschl G. The oscillating central network of essential tremor. Clinical 590 neurophysiology. 2012;123(1):61-64.

[21] Pavlides A, Hogan SJ, Bogacz R. Computational models describing possible mechanisms for ${ }_{592}$ generation of excessive beta oscillations in Parkinson's disease. PLoS computational biology. 593 2015;11(12):e1004609.

[22] Kuramoto Y, Araki H. Proceedings of the International Symposium on Mathematical Problems in Theoretical Physics; 1975.

[23] Acebrón JA, Bonilla LL, Vicente CJP, Ritort F, Spigler R. The Kuramoto model: A simple paradigm for synchronization phenomena. Reviews of modern physics. 2005;77(1):137.

[24] Stiefel KM, Gutkin BS, Sejnowski TJ. Cholinergic neuromodulation changes phase response curve shape and type in cortical pyramidal neurons. PloS one. 2008;3(12):e3947.

[25] Fong R, Russell J, Weerasinghe G, Bogacz R. Kuramoto Model Simulation; 2018. University of Oxford, doi:10.5287/bodleian:6qJdGNDPj, available at: https://data.mrc.ox.ac.uk/dataset/kuramoto.

[26] Ott E, Antonsen TM. Low dimensional behavior of large systems of globally coupled oscillators. Chaos: An Interdisciplinary Journal of Nonlinear Science. 2008;18(3):037113.

[27] Bick C, Goodfellow M, Laing CR, Martens EA. Understanding the dynamics of biological and neural oscillator networks through exact mean-field reductions: a review. The Journal of Mathematical Neuroscience. 2020;10(1):1-43.

[28] Duchet B, Weerasinghe G, Cagnan H, Brown P, Bick C, Bogacz R. Phase-dependence of response curves to deep brain stimulation and their relationship: from essential tremor patient data to a Wilson-Cowan model. The Journal of Mathematical Neuroscience. 2020;10(1):1-39.

[29] Cagnan H, Weerasinghe G, Brown P. Tremor data measured from essential tremor patients subjected to phase-locked deep brain stimulation; 2019. University of Oxford, doi:10.5287/bodleian:xq24eN2Km, available at: https://data.mrc.ox.ac.uk/data-set/tremordata-measured-essential-tremor-patients-subjected-phase-locked-deep-brain.

[30] Goetz CG, Tilley BC, Shaftman SR, Stebbins GT, Fahn S, Martinez-Martin P, et al. Movement Disorder Society-sponsored revision of the Unified Parkinson's Disease Rating Scale (MDSUPDRS): scale presentation and clinimetric testing results. Movement disorders: official journal of the Movement Disorder Society. 2008;23(15):2129-2170.

[31] Hagen E, Næss S, Ness TV, Einevoll GT. Multimodal modeling of neural network activity: computing LFP, ECoG, EEG, and MEG signals with LFPy 2.0. Frontiers in neuroinformatics. $2018 ; 12: 92$.

[32] Naik GR, Kumar DK. An overview of independent component analysis and its applications. Informatica. 2011;35(1).

[33] Tass PA, Majtanik M. Long-term anti-kindling effects of desynchronizing brain stimulation: a theoretical study. Biological cybernetics. 2006;94(1):58-66.

[34] Hofmanis J, Ruiz RAS, Caspary O, Ranta R, Louis-Dorr V. Extraction of Deep Brain Stimulation (DBS) source in SEEG using EMD and ICA. In: 2011 Annual International Conference of the IEEE Engineering in Medicine and Biology Society. IEEE; 2011. p. 834-837.

[35] Abbasi O, Hirschmann J, Schmitz G, Schnitzler A, Butz M. Rejecting deep brain stimulation artefacts from MEG data using ICA and mutual information. Journal of neuroscience methods. 2016;268:131-141.

[36] Oswal A, Jha A, Neal S, Reid A, Bradbury D, Aston P, et al. Analysis of simultaneous MEG ${ }^{633}$ and intracranial LFP recordings during Deep Brain Stimulation: a protocol and experimental 634 validation. Journal of neuroscience methods. 2016;261:29-46.

[37] Lagarias JC, Reeds JA, Wright MH, Wright PE. Convergence properties of the Nelder-Mead 636 simplex method in low dimensions. SIAM Journal on optimization. 1998;9(1):112-147. 